\title{
Variable Sodium Absorption in a Low-extinction Type la Supernova,
}

\section{Citation}

Simon, Joshua D., Avishay Gal-Yam, Orly Gnat, Robert M. Quimby, Mohan Ganeshalingam, Jeffrey M. Silverman, Stephane Blondin, et al. 2009. "VARIABLE SODIUM ABSORPTION IN A LOW-EXTINCTION TYPE la SUPERNOVA,." The Astrophysical Journal 702 (2): 1157-70. https:// doi.org/10.1088/0004-637x/702/2/1157.

\section{Permanent link}

http://nrs.harvard.edu/urn-3:HUL.InstRepos:41399752

\section{Terms of Use}

This article was downloaded from Harvard University's DASH repository, and is made available under the terms and conditions applicable to Other Posted Material, as set forth at http:// nrs.harvard.edu/urn-3:HUL.InstRepos:dash.current.terms-of-use\#LAA

\section{Share Your Story}

The Harvard community has made this article openly available.

Please share how this access benefits you. Submit a story.

Accessibility 


\title{
VARIABLE SODIUM ABSORPTION IN A LOW-EXTINCTION TYPE Ia SUPERNOVA*,
}

\author{
Joshua D. Simon ${ }^{1}$, Avishay Gal-Yam ${ }^{2}$, Orly Gnat ${ }^{3,18}$, Robert M. Quimby ${ }^{4}$, Mohan Ganeshalingam ${ }^{5}$, JefFrey $^{2}$ \\ M. Silverman ${ }^{5}$, Stephane Blondin $^{6}$, Weidong Li $^{5}$, Alexei V. Filippenko ${ }^{5}$, J. Craig Wheeler ${ }^{7}$, Robert P. Kirshner ${ }^{8}$, \\ Ferdinando Patat ${ }^{6}$, Peter Nugent ${ }^{9}$, Ryan J. Foley ${ }^{8,19}$, Steven S. Vogt ${ }^{10}$, R. Paul Butler ${ }^{11}$, Kathryn M. G. Peek ${ }^{5}$, \\ Erik Rosolowsky ${ }^{12}$, Gregory J. Herczeg ${ }^{13}$, Daniel N. Sauer ${ }^{14}$, and Paolo A. Mazzali ${ }^{15,16,17}$ \\ ${ }^{1}$ Observatories of the Carnegie Institution of Washington, 813 Santa Barbara Street, Pasadena, CA 91101, USA; jsimon@ociw.edu \\ ${ }^{2}$ Benoziyo Center for Astrophysics, Faculty of Physics, Weizmann Institute of Science, 76100 Rehovot, Israel; avishay.gal-yam@weizmann.ac.il \\ ${ }^{3}$ Theoretical Astrophysics, California Institute of Technology, Mail Code 130-33, 1200 E. California Boulevard, Pasadena, CA 91125, USA; orlyg@tapir.caltech.edu \\ ${ }^{4}$ Department of Astronomy, California Institute of Technology, 1200 E. California Boulevard, MS 105-24, Pasadena, CA 91125, USA; quimby@astro.caltech.edu \\ ${ }^{5}$ Department of Astronomy, University of California, Berkeley, CA 94720-3411, USA; mganesh@astro.berkeley.edu, jsilverman@astro.berkeley.edu, \\ weidong@astro.berkeley.edu, alex@astro.berkeley.edu,kpeek@astro.berkeley.edu \\ ${ }^{6}$ European Southern Observatory, Karl Schwarzschild Str. 2, D-85748 Garching bei München, Germany; sblondin@eso.org, fpatat@eso.org \\ ${ }^{7}$ McDonald Observatory and Department of Astronomy, University of Texas, Austin, TX 71782, USA; wheel@ astro.as.utexas.edu \\ ${ }^{8}$ Harvard-Smithsonian Center for Astrophysics, 60 Garden Street, Cambridge, MA 02138, USA; kirshner@cfa.harvard.edu, rfoley@cfa.harvard.edu \\ ${ }^{9}$ Lawrence Berkeley National Laboratory, 1 Cyclotron Road, Berkeley, CA 94720, USA; penugent@1bl.gov \\ ${ }^{10}$ UCO/Lick Observatory, University of California, Santa Cruz, CA 95064, USA; vogt@ucolick.org \\ ${ }^{11}$ Department of Terrestrial Magnetism, Carnegie Institution of Washington, 5241 Broad Branch Road NW, Washington, DC 20015, USA; butler@dtm.ciw.edu \\ ${ }^{12}$ Department of Physics, University of British Columbia, Okanagan, BC V1V 1V7, Canada; erik.rosolowsky@ubc.ca \\ ${ }^{13}$ Max-Planck-Institut für Extraterrestiche Physik, Postfach 1312, 85741 Garching, Germany; gregoryh@mpe.mpg.de \\ ${ }^{14}$ Department of Astronomy, Stockholm University, 10691 Stockholm, Sweden; dsauer@astro.su.se \\ ${ }^{15}$ Max-Planck-Institut für Astrophysik, Karl-Schwarzschild-Strasse 1, 85741 Garching, Germany; mazzali@mpa-garching.mpg.de \\ ${ }^{16}$ Scuola Normale Superiore, Piazza dei Cavalieri 7, 56126 Pisa, Italy \\ ${ }^{17}$ INAF-Osservatorio Astronomico di Padova, Vicolo dell'Osservatorio 5, 35122 Padova, Italy \\ Received 2009 May 1; accepted 2009 July 15; published 2009 August 19
}

\begin{abstract}
Recent observations have revealed that some Type Ia supernovae exhibit narrow, time-variable Na I D absorption features. The origin of the absorbing material is controversial, but it may suggest the presence of circumstellar gas in the progenitor system prior to the explosion, with significant implications for the nature of the supernova (SN) progenitors. We present the third detection of such variable absorption, based on six epochs of high-resolution spectroscopy of the Type Ia supernova SN 2007le from the Keck I Telescope and the Hobby-Eberly Telescope. The data span a time frame of approximately three months, from 5 days before maximum light to 90 days after maximum. We find that one component of the $\mathrm{Na}$ I $\mathrm{D}$ absorption lines strengthened significantly with time, indicating a total column density increase of $\sim 2.5 \times 10^{12} \mathrm{~cm}^{-2}$. The data limit the typical timescale for the variability to be more than 2 days but less than 10 days. The changes appear to be most prominent after maximum light rather than at earlier times when the ultraviolet flux from the SN peaks. As with SN 2006X, we detect no change in the $\mathrm{Ca}$ II $\mathrm{H}$ and $\mathrm{K}$ absorption lines over the same time period, rendering line-of-sight effects improbable and suggesting a circumstellar origin for the absorbing material. Unlike the previous two supernovae exhibiting variable absorption, SN 2007le is not highly reddened $\left(E_{B-V}=0.27 \mathrm{mag}\right)$, also pointing toward circumstellar rather than interstellar absorption. Photoionization calculations show that the data are consistent with a dense $\left(10^{7} \mathrm{~cm}^{-3}\right)$ cloud or clouds of gas located $\sim 0.1 \mathrm{pc}\left(3 \times 10^{17} \mathrm{~cm}\right)$ from the explosion. These results broadly support the singledegenerate scenario previously proposed to explain the variable absorption, with mass loss from a nondegenerate companion star responsible for providing the circumstellar gas. We also present possible evidence for narrow $\mathrm{H} \alpha$ emission associated with the SN, which will require deep imaging and spectroscopy at late times to confirm.
\end{abstract}

Key words: circumstellar matter - supernovae: general - supernovae: individual (SN 1999cl, SN 2006X, SN 2007le)

Online-only material: color figures

\section{INTRODUCTION}

The origin of Type Ia supernovae (SNe Ia) has important implications for understanding the physics of these dramatic

\footnotetext{
* Some of the data presented herein were obtained at the W. M. Keck Observatory, which is operated as a scientific partnership among the California Institute of Technology, the University of California, and NASA. The Observatory was made possible by the generous financial support of the W. M. Keck Foundation.

$\dagger$ Based in part on observations obtained with the Hobby-Eberly Telescope, which is a joint project of the University of Texas at Austin, the Pennsylvania State University, Stanford University, Ludwig-Maximilians-Universität München, and Georg-August-Universität Göttingen.

18 Chandra Fellow.

${ }^{19}$ Clay Fellow.
}

explosions, the evolution of binary stars, and the expansion of the universe. Recent interest in this last issue is particularly high because $\mathrm{SNe}$ Ia are the most effective distance indicators known on cosmological scales. These objects are thus the focus of many current and future dark energy experiments (e.g., Astier et al. 2006; Riess et al. 2007; Wood-Vasey et al. 2007; Aldering 2005). Investigating systematics that could affect their luminosity evolution in any way is a prerequisite for precision cosmological studies using SNe Ia as calibratable standard candles.

Perhaps the most serious gap in our understanding of $\mathrm{SNe}$ Ia is our lack of knowledge about their progenitor systems. It is widely thought that $\mathrm{SNe}$ Ia occur in close binary systems 
(Whelan \& Iben 1973), in which a carbon-oxygen white dwarf accretes matter from another star until it nears the Chandrasekhar (1931) mass and its core ignites a thermonuclear explosion that propagates outward and destroys the white dwarf. The identity of the companion star, however, is unknown; possibilities range from a second white dwarf (a doubledegenerate system; Iben \& Tutukov 1984; Webbink 1984) to a main-sequence star, subgiant, or evolved red giant (all singledegenerate systems). At best, these proposed progenitor systems would have an absolute magnitude of $M_{V} \approx-3$ (for a red giant companion star near the tip of the red giant branch), leaving them undetectable by current telescopes for all but the closest galaxies. Since the last known Local Group SN Ia occurred in 1885 (de Vaucouleurs \& Corwin 1985; Fesen et al. 1989) and there have been no SNe Ia observed in the Milky Way in over 400 years (Ruiz-Lapuente 2004; Badenes et al. 2006; Krause et al. 2008), direct empirical constraints on the nature of the progenitors are severely lacking.

Recent observations, however, have yielded progress on this issue using several different approaches. First, statistical studies of large numbers of supernova (SN) host galaxies have demonstrated a strong relationship between Hubble type and the rate of $\mathrm{SNe} \mathrm{Ia}$, with late-type galaxies hosting as many as 20 times more SNe Ia per unit mass as early-type galaxies (e.g., Oemler \& Tinsley 1979; Mannucci et al. 2005; Sullivan et al. 2006). This result suggests that many SNe Ia come from a relatively young stellar population, although of course the $\mathrm{SNe} \mathrm{Ia}$ in elliptical galaxies must have their origin in an older population. These observations, combined with the SN Ia rate as a function of redshift, can be used to derive the delay-time distribution (the amount of time between the formation of a stellar population and the occurrence of $\mathrm{SNe}$ Ia). The measured delay-time distribution includes both a prompt $\left(\sim 10^{8} \mathrm{yr}\right)$ component that is closely associated with recent star formation and a much more extended component with a characteristic timescale of several Gyr (Scannapieco \& Bildsten 2005; Mannucci et al. 2006, although see Totani et al. 2008 for a conflicting view). These two distinct components of the SN Ia population would seem to naturally correspond to different mechanisms for producing SNe Ia, and perhaps different progenitor systems as well. Supporting this idea, a number of studies have demonstrated that SNe Ia in early-type and late-type galaxies have different average luminosities (e.g., Hamuy et al. 1996; Howell 2001; Li et al. 2001; Hicken et al. 2009).

Detailed studies of individual nearby $\mathrm{SNe}$ Ia have also revealed crucial clues. Quimby et al. (2007) found unusual behavior of the velocity of the Si II $\lambda 6355$ line in the otherwise normal SN 2005hj and argued that this object might be the prototype of a separate class of SN Ia explosions. Ruiz-Lapuente et al. (2004) obtained spectra of stars near the center of the remnant of Tycho's supernova (SN 1572) and claimed to identify the companion star to the SN progenitor, a G-type subgiant, although this interpretation has proved controversial (e.g., Ihara et al. 2007; González-Hernández et al. 2009; Kerzendorf et al. 2009).

Finally, another recent advance has been made by Patat et al. (2007a), who detected circumstellar material (CSM) in a SN Ia via optical absorption lines for the first time. Previous searches for emission from CSM in SNe Ia using $\mathrm{H} \alpha$, radio, and X-ray observations have generally yielded only upper limits (Mattila et al. 2005; Leonard 2007; Panagia et al. 2006; Immler et al. 2006; Hughes et al. 2007), with the exception of a few unusual objects whose classification as $\mathrm{SNe}$ Ia has been disputed (e.g., Li et al. 2001, 2003; Hamuy et al. 2003; Aldering et al. 2006; Prieto et al. 2007, but see Benetti et al. 2006 and Trundle et al. 2008 for alternative interpretations). By obtaining high-resolution spectra of SN 2006X at multiple epochs, Patat et al. showed that the strength of at least four distinct components of the $\mathrm{Na}$ I $\mathrm{D}$ absorption lines varied with time. The temporal coverage of the data was rather sparse, but the variability occurred over the time span between 2 days before maximum light and 61 days after. Similar rapid changes in metal absorption-line profiles with time are commonly seen in novae and provide clues to the mass-transfer process in those systems (Williams et al. 2008). Variable Na D absorption is also observed in the spectra of Milky Way stars (generally on timescales of years to decades, although observations on shorter timescales are lacking) and is usually attributed to small interstellar clouds moving perpendicular to the line of sight (e.g., Welty \& Fitzpatrick 2001). The lack of corresponding variability in the $\mathrm{Ca}$ II $\mathrm{H} \& \mathrm{~K}$ absorption features in SN 2006X, however, renders this interpretation viable only with an appeal to peculiar chemical abundances and/or an unusual geometry in the absorbing interstellar clouds.

Accordingly, Patat et al. (2007a) concluded that the variable absorption features originate in circumstellar clouds in the progenitor system that were ionized by the radiation from the SN and recombined over the following several weeks, with some of the clouds then being collisionally reionized by the SN ejecta at later times. This circumstellar gas could have originated either in the stellar wind from the progenitor's companion star or in successive nova eruptions. Because $\mathrm{Na}$ I has a much lower ionization potential than $\mathrm{Ca}$ II, the $\mathrm{Na} \mathrm{D}$ line profiles can change without an accompanying effect in the $\mathrm{Ca} \mathrm{H} \& \mathrm{~K}$ lines if the ionizing radiation has an appropriate spectrum. The Patat et al. interpretation has been challenged by Chugai (2008), who showed that for typical red giant wind densities $\mathrm{Na} \mathrm{D}$ absorption should not be detectable. Chugai (2008) suggested instead that the absorbing material must be located farther away from the $\mathrm{SN}$, possibly not associated with the progenitor system at all. Photometry and late-time low-resolution spectroscopy of SN 2006X, which reveal at least one light echo from the SN, also provide support for a dusty circumstellar environment (Wang et al. 2008a, 2008b; Crotts \& Yourdon 2008). Taken as a whole, these results appear to indicate a single-degenerate progenitor for SN 2006X, perhaps with a red giant companion.

But is SN 2006X merely a unique, pathological object? Or is it broadly representative of a significant fraction of (or perhaps all) SNe Ia? Blondin et al. (2009) have now identified a second $\mathrm{SN}, \mathrm{SN}$ 1999cl, displaying variable $\mathrm{Na} \mathrm{D}$ absorption that is visible even at low spectral resolution, but the lack of highresolution data or coverage of the $\mathrm{Ca} H$ \& K lines makes the interpretation of this object more difficult. The Blondin et al. analysis demonstrates that significant changes in the $\mathrm{Na}$ $\mathrm{D}$ absorption profile are relatively uncommon, with only two detections out of $31 \mathrm{SNe}$ examined. Notably, SN 1999cl and SN 2006X are the two most heavily reddened SNe in the Blondin et al. (2009) sample, creating the appearance of a connection between large (presumably interstellar) reddening and variable absorption.

Only two other SNe Ia (SN 2000cx and SN 2007af) have multi-epoch high-resolution spectra available in the literature, and neither of those objects exhibits any changes in the $\mathrm{Na} \mathrm{D}$ line profiles with time (Patat et al. 2007b; Simon et al. 2007). In this paper, we present a similar data set for the bright SN Ia 2007le, including six high-resolution spectra obtained between -5 and 
Table 1

High-Resolution Spectroscopy Observing Log

\begin{tabular}{|c|c|c|c|c|c|c|c|}
\hline Telescope & Instrument & UT Date & Supernova Epoch ${ }^{\mathrm{a}}$ & $\begin{array}{c}\text { Exposure Time } \\
(\mathrm{s})\end{array}$ & $\mathrm{S} / \mathrm{N}^{\mathrm{b}}$ & $\begin{array}{c}\text { Spectral Resolution }^{\mathrm{c}} \\
(\lambda / \Delta \lambda)\end{array}$ & $\begin{array}{c}\text { Wavelength Range } \\
(\AA)\end{array}$ \\
\hline Keck I & HIRESb & 2007 October 20.36 & day -5 & 7200 & 113 & 52,000 & $3840-6733$ \\
\hline Keck I & HIRESr & 2007 October 25.34 & day 0 & 900 & 71 & 41,700 & $3460-7989$ \\
\hline HET & HRS & 2007 November 4.15 & day +10 & 3000 & 16 & 60,000 & $5100-8850$ \\
\hline Keck I & HIRESb & 2007 November 6.39 & day +12 & 4500 & 76 & 47,600 & $3845-6649$ \\
\hline Keck I & HIRESr & 2008 January 17.23 & day +84 & 3300 & 46 & 54,000 & $3870-8365$ \\
\hline Keck I & HIRESr & 2008 January 23.21 & day +90 & 1200 & 11 & 54,000 & $3900-8360$ \\
\hline
\end{tabular}

Notes.

${ }^{\text {a }}$ Relative to maximum light.

b Signal-to-noise ratio measurements are per pixel at the wavelength of the redshifted Na D lines (5934.5-5936.5 $\mathrm{A})$.

c The spectral resolution was determined by measuring the FWHM of emission lines in the comparison-lamp spectra and telluric absorption lines in the SN and telluric standard star spectra.

+90 days relative to maximum light. We use these data to test the Patat et al. (2007a) scenario, searching for variability in the $\mathrm{Na} \mathrm{D}$ absorption features. We describe all of our observations, both photometric and spectroscopic, in Section 2. In Section 3, we compare the light curve and spectral evolution of SN 2007le with those of other SNe Ia and analyze the high-resolution spectra. We discuss the implications of the variable $\mathrm{Na}$ D absorption features in Section 4 and present our conclusions in Section 5.

\section{OBSERVATIONS AND DATA REDUCTION}

SN 2007le was discovered by L. Monard (Monard et al. 2007) on 2007 October 13.79 (UT dates are used throughout this paper). Optical spectra obtained two nights later showed that the object was a SN Ia at least one week before maximum light, and featuring high-velocity ejecta expanding at $\sim 16,000 \mathrm{~km} \mathrm{~s}^{-1}$ (Filippenko et al. 2007). The host galaxy of the SN is NGC 7721, an Sc galaxy with a heliocentric recession velocity of $2015 \mathrm{~km} \mathrm{~s}^{-1}$ (Koribalski et al. 2004).

\subsection{High-resolution Spectroscopy}

Our high-resolution observing campaign for SN 2007le began on 2007 October 20 with the High Resolution Echelle Spectrometer (HIRES; Vogt et al. 1994) on the Keck I $10 \mathrm{~m}$ telescope. Over the following three months, we obtained a total of five HIRES spectra of the SN. The seeing was often poor $\left(\gtrsim 1^{\prime \prime}\right)$ during the observations, and the overall conditions varied significantly over the course of the many observing runs. The data were obtained with a range of different spectrograph setups, including spectra with both the blue and red cross-dispersers, and achieved signal-to-noise ratios $(\mathrm{S} / \mathrm{Ns})$ ranging between 11 and 113 per pixel. Most of the spectra used a 7". $0 \times 0$ 0'.86 slit, yielding a spectral resolution of $R \approx 52,000$, but one spectrum was obtained with a wider $\left(7^{\prime \prime} .0 \times 11^{\prime \prime} .15\right)$ slit and $R \approx 42,000$. All of the HIRES spectra cover the $\mathrm{Ca} H \& \mathrm{~K}, \mathrm{Na}$, and $\mathrm{H} \alpha$ lines. We reduced the Keck data using the IDL data reduction package for HIRES (version 2.0) developed by J. X. Prochaska and collaborators (R. Bernstein et al. 2009, in preparation). ${ }^{20}$

We also obtained one spectrum of SN 2007le with the HighResolution Spectrograph (HRS; Tull 1998) on the HobbyEberly Telescope (HET) on 2007 November 4 . The spectrograph was in its $R=60,000$ mode, with a $2^{\prime \prime}$-diameter fiber and the 316 line/mm grating centered at $6948 \AA$, providing nearly complete wavelength coverage from 5100 to $8850 \AA$. We

\footnotetext{
${ }^{20}$ Documentation and code for this reduction package are available at http://www.ucolick.org/ xavier/HIRedux/index.html.
}

obtained 3 spectra totaling $3000 \mathrm{~s}$ of exposure time and reached a combined $\mathrm{S} / \mathrm{N}$ of 16 per pixel. The HRS data were reduced in IRAF ${ }^{21}$ with the ECHELLE package using standard procedures. A comprehensive summary of all the high-resolution data is presented in Table 1.

\subsection{Imaging and Low-resolution Spectroscopy}

SN 2007le was the target of extensive photometric followup observations with the $0.76 \mathrm{~m}$ Katzman Automatic Imaging Telescope (KAIT; Li et al. 2000; Filippenko et al. 2001), continuing for approximately three months until the SN went into conjunction with the Sun. We obtained post-explosion images in 2008 August after the SN had faded in order to subtract the host-galaxy light. We used the DAOPHOT package (Stetson 1987) in IRAF to perform point-spread function photometry of SN 2007le relative to various field stars in the KAIT images, which were calibrated on five photometric nights with KAIT and the Nickel $1 \mathrm{~m}$ telescope at Lick Observatory.

We also obtained low-resolution spectra of SN 2007le with the Low Resolution Imaging Spectrometer (LRIS; Oke et al. 1995) on the Keck I telescope on 2007 October 15, October 16, November 11, November 12, and December 12, and with the Kast spectrograph (Miller \& Stone 1993) on the $3 \mathrm{~m}$ Shane telescope at Lick Observatory on 2007 November 2, November 18, and December 1. These data were reduced in IRAF and IDL following normal procedures (for details, see Foley et al. 2003; Matheson et al. 2000; Horne 1986).

\section{DATA ANALYSIS AND RESULTS}

\subsection{Light Curves and Low-resolution Spectra}

We display BVRI light curves of SN 2007le in Figure 1. We fitted the photometric data with the latest version of the multicolor light curve shape method (MLCS2k2; Jha et al. 2007) to determine the parameters of the $\mathrm{SN}$. We find that the time of $B$ band maximum was 2007 October 25.65 (JD = 2,454,399.15), with an uncertainty of 0.09 day. The derived line-of-sight extinction to the $\mathrm{SN}$ is $A_{V}=0.71 \pm 0.06 \mathrm{mag}$, with an extinction law of $R_{V}=2.56 \pm 0.22$ (the Milky Way foreground reddening is $0.033 \mathrm{mag}$, corresponding to $A_{V}=0.11 \mathrm{mag}$; Schlegel et al. 1998). The distance modulus to SN 2007le is $m-M=(32.35-$

${ }^{21}$ IRAF is distributed by the National Optical Astronomy Observatories, which are operated by the Association of Universities for Research in Astronomy, Inc., under cooperative agreement with the National Science Foundation. 


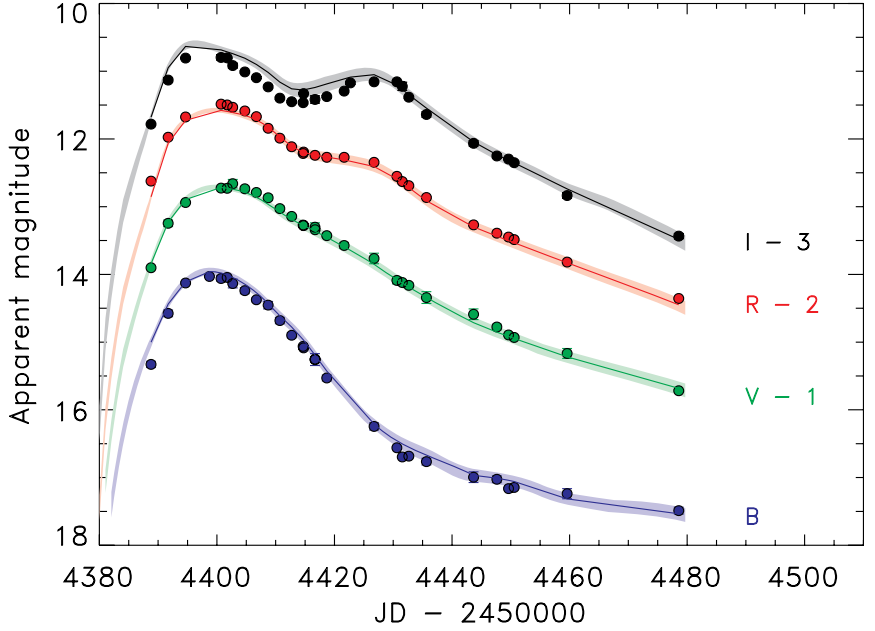

Figure 1. BVRI light curves (filled symbols) of SN 2007le, from KAIT and Lick Nickel $1 \mathrm{~m}$ data. Photometric uncertainties are indicated by the plotted error bars, which in most cases are smaller than the displayed data points. The solid lines represent the MLCS2k2 fits to the data and the shaded bands represent the uncertainties on the fits.

(A color version of this figure is available in the online journal.)

$\left.5 \log \left[\mathrm{H}_{0} /\left(70 \mathrm{~km} \mathrm{~s}^{-1} \mathrm{Mpc}^{-1}\right)\right]\right) \pm 0.06 \mathrm{mag}$, giving the $\mathrm{SN}$ an absolute magnitude of $M_{V}=\left(-19.34+5 \log \mathrm{H}_{0} / 70\right) \pm 0.09$. The luminosity/light curve-shape parameter is $\Delta=-0.14 \pm 0.02$, and the MLCS2k $2 \chi^{2}$ value of 163.0 for 106 degrees of freedom indicates an acceptable fit (much of the $\chi^{2}$ comes from the $I$-band data, which significantly differ from the fit).

In Figure 2, we show the spectrum of SN 2007le at an epoch of 10.3 days before maximum light. Analysis with the Superfit spectral fitting code of Howell et al. (2005) indicates that SN 2007le is most similar to spectra of the highly reddened type Ia SN 2002bo at 11 days before maximum from Benetti et al. (2004), while the SNID package (Blondin \& Tonry 2007) finds a best fit to early spectra of the "golden standard" type Ia SN 2005cf (Wang et al. 2009). Spectra of SN 2002bo and SN 2005cf were included in the databases used by both fitting packages; the differences between the two lie in their fitting methods and treatment of color information (see Blondin \& Tonry 2007), but since SN 2002bo and SN 2005cf were similar events in many respects (e.g., line velocities) we do not regard these fit results as a significant disagreement. Like SN 2002bo (and SN 2006X; Wang et al. 2008a), SN 2007le exhibits a high-velocity component to the $\mathrm{Ca}$ II near-infrared triplet absorption lines, although such features are very common in SNe Ia that are observed at sufficiently early times (Mazzali et al. 2005b). Both SN 2006X and SN 2002bo were highly polarized (Wang et al. 2006, 2007; Wang \& Wheeler 2008), suggesting that polarization measurements of SN 2007le might be very interesting as well.

In order to classify SN 2007le among the various known subgroups of SNe Ia, we analyzed the spectra according to the prescriptions of Benetti et al. (2005) and Branch et al. (2006, 2009). The widths of the absorption features near $5750 \AA$ and $6100 \AA$ correspond to the broad line (BL) group of Branch et al. but the equivalent widths are not too far from those of the core normal group. We measured a velocity gradient in the Si II $\lambda 6355$ line of $83 \pm 3 \mathrm{~km} \mathrm{~s}^{-1} \mathrm{day}^{-1}$, placing SN 2007le marginally within the high velocity gradient (HVG) class of Benetti et al. (2005), although this gradient is not very much larger than is seen in some low velocity gradient SNe. SN 2006X and SN 2002 bo both exhibited significantly larger gradients.

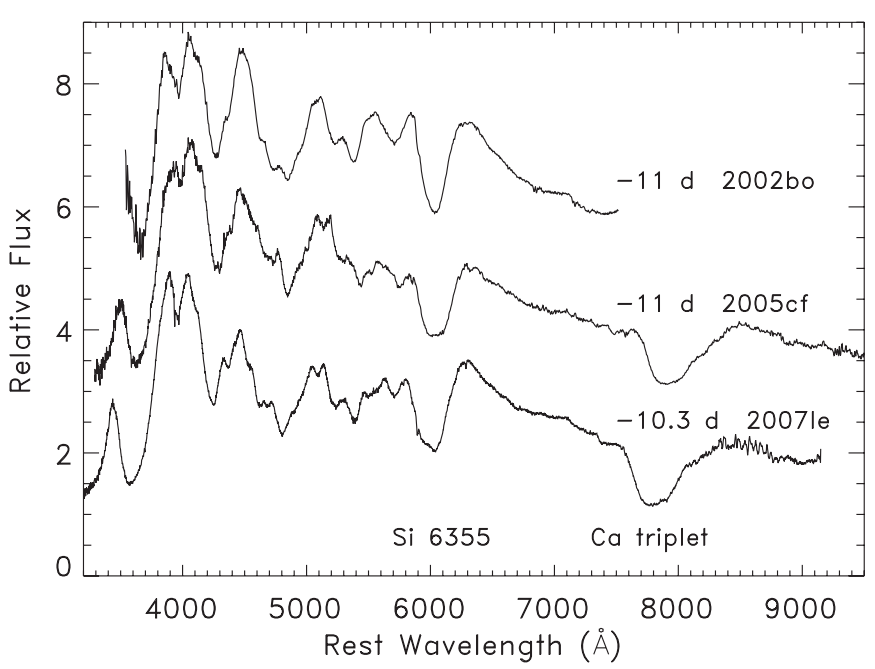

Figure 2. Low-resolution spectrum of SN 2007le 10.3 days before maximum light (lower curve), compared to scaled spectra of SN 2005cf (middle, data from Wang et al. 2009) and SN 2002bo (top, data from Benetti et al. 2004) at similar epochs. The spectral features match closely between the three supernovae. The SN 2005cf and SN 2002bo data have been dereddened by $A_{V}=0.58 \mathrm{mag}$ (Wang et al. 2009) and $A_{V}=1.0 \mathrm{mag}$ (Elias-Rosa et al. 2008), respectively, and then re-reddened by $A_{V}=0.71 \mathrm{mag}$ to match the extinction of SN 2007le.

\subsection{Removal of Telluric Absorption Features}

The host-galaxy Na D absorption lines of SN 2007le are located at observed wavelengths between 5930 and $5940 \AA$. This region of the spectrum unfortunately contains a number of telluric absorption lines from water molecules (e.g., Moore et al. 1966). Before searching for variations in the intrinsic SN line profiles, we therefore must remove the telluric features, which will change with atmospheric conditions (e.g., Wade \& Horne 1988; Matheson et al. 2001).

On every night except that of the second-epoch observations (day 0, 2007 October 25), we obtained at least one spectrum of a hot, rapidly rotating star to serve as a telluric standard. These spectra were reduced and normalized to a flat continuum level in the same manner as the SN data. Because the telluric standards do not contain any atmospheric features in the region of interest, any absorption seen must necessarily be of telluric origin. From the telluric standard observations we constructed telluric absorption line model spectra using a list of known telluric lines. ${ }^{22}$ Pixels in the model spectra that were at the wavelength of telluric lines were set equal to the value of those pixels in the telluric standard star spectra, and all other pixels were set to unity. We then selected the telluric model obtained at the most similar airmass and time for each SN spectrum. ${ }^{23}$ The telluric models were further adjusted to match the air mass of the SN observations with the following scaling: model $_{\text {adjusted }}=$ $\left(\text { model }_{\text {original }}\right)^{b}$, where $b=$ air mass $\operatorname{sN}_{S} /$ air mass standard $_{\text {. Finally, }}$ we divided the reduced SN spectra by their respective telluric absorption models to produce clean SN spectra. The strength of the absorption lines that overlap with the wavelength of the host-galaxy $\mathrm{Na} \mathrm{D}$ absorption in the telluric models ranges from

\footnotetext{
22 We use a model rather than simply the observed standard star spectrum in order to remove any effects from imperfectly fitting the continuum of the standard star and because the $\mathrm{S} / \mathrm{N}$ of the standard spectra is in some cases not too much higher than the $\mathrm{S} / \mathrm{N}$ of the $\mathrm{SN}$ spectrum.

23 For the second-epoch observations where we lack a telluric standard from the same night, we use a telluric standard from the night of 2007 October 20 , which had the most similar atmospheric water vapor content to 2007 October 25 according to the Mauna Kea Weather Center forecasts (available on the internet) for the nights on which we observed.
} 


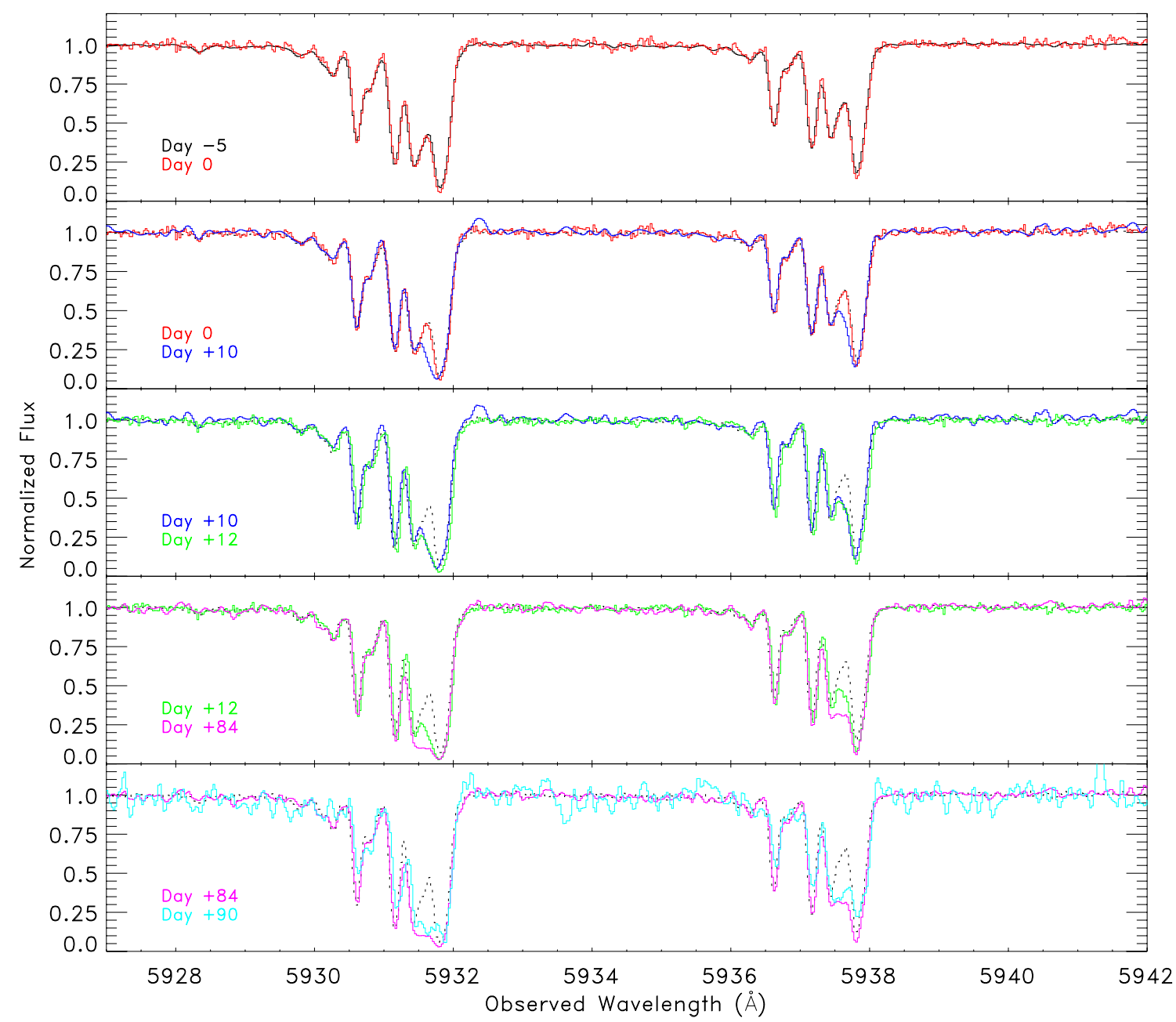

Figure 3. High-resolution observations of the Na D absorption lines in the spectrum of SN 2007le near the host-galaxy velocity. The top panel shows the initial HIRES spectrum (day -5) in black and the second-epoch spectrum from day 0 later in red. In each subsequent panel the initial spectrum is plotted as a dotted black curve. The second panel compares the day 0 (red) and day +10 (blue) spectra to each other, and then each following panel does the same for the next neighboring pair of observations. All of the spectra shown in each panel are smoothed to the lowest resolution spectrum displayed in that panel (which means that the effective resolution changes somewhat from panel to panel). At least one component of the $\mathrm{Na}$ absorption near $5931.5 \AA$ in the $\mathrm{D}_{2}$ line clearly strengthens with time.

a few percent to $10 \%$, not nearly large enough to account for the absorption profile changes described in Section 3.3.

\subsection{Sodium D Absorption Lines at High Resolution}

We display the high-resolution spectra of SN 2007le around the host-galaxy $\mathrm{Na} \mathrm{D}$ lines in Figure 3. At least one component of the absorption (near $5931.5 \AA$ and $5937.5 \AA$ in the $\mathrm{D}_{2}$ and $\mathrm{D}_{1}$ lines, respectively) strengthens over the course of the observations, increasing from a total depth of $\sim 0.6$ in the first spectrum to nearly saturated three months later. ${ }^{24}$ The full absorption profile is complex, with $\sim 7$ distinct velocity components visible. Fitting the absorption with a set of Gaussians reveals that at least two additional components blended with the strongest absorption features are needed in order to remove significant residuals, and even then the fits to the high-S/N spectra are not statistically

\footnotetext{
${ }^{24}$ In Figure 3, it appears that the final (day +90) spectrum shows additional changes, with all of the $\mathrm{Na}$ absorption features weakening compared to the previous epochs. Given the complete lack of variations in all but one of these components through the day +84 spectrum obtained just six days earlier, the very poor observing conditions under which the day +90 spectrum was acquired (bad seeing, high humidity, and bright sky because the SN set before the end of twilight), and the low $\mathrm{S} / \mathrm{N}$ of the spectrum, we suspect that these changes indicate a systematic problem with the data rather than true physical changes. We therefore exclude the day +90 spectrum from the remainder of our analysis.
}

satisfactory. Fits of multiple blended Gaussians do not produce unique results, however, so we cannot study the physical conditions in the variable absorption component(s) accurately with this technique.

Instead, wefirst integrate directly over the entire absorption profile to determine the equivalent width (EW) of the $\mathrm{D}_{1}$ and $\mathrm{D}_{2}$ lines in each spectrum. The measured equivalent widths are listed in columns (2) and (3) of Table 2. Over the course of the observations, both lines show an increase in the EW of slightly more than $100 \mathrm{~mA}$. The total EWs in the day -5 spectrum are $894 \pm 2 \mathrm{~m} \AA$ and $649 \pm 2 \mathrm{~m} \AA$ for the $\mathrm{D}_{2}$ and $\mathrm{D}_{1}$ lines, respectively. By the final spectrum at day +84 , the EWs are $1006 \pm 5 \mathrm{~m} \AA$ and $766 \pm 5 \mathrm{~m} \AA$. For the four blue most components of the absorption, we rule out any variation at high significance; the EWs of those components agree in all six epochs to a level of a few $m \AA$. To reduce the uncertainty in the estimate of the change in EW, we can therefore leave these components out of the integration and sum the absorption starting from the peak at $5931 \AA$ (5937 $\AA$ for the $D_{1}$ line) instead of from the blue edge of the absorption at $5929.5 \AA$ (5935.5 $\AA$ ). With this smaller wavelength range we find a total increase in $\mathrm{EW}$ between day -5 and day +84 of $106 \pm 5 \mathrm{~m} \AA$ and $105 \pm 5 \mathrm{~m} \AA$ for the $\mathrm{D}_{2}$ and $\mathrm{D}_{1}$ lines (last two columns of Table 2). 
Table 2

Na D Equivalent Widths

\begin{tabular}{lccccc}
\hline \hline \multirow{2}{*}{ Epoch } & \multicolumn{2}{c}{ Full Profile } & & \multicolumn{2}{c}{ Red Side } \\
\cline { 2 - 3 } & $\begin{array}{c}\mathrm{D}_{2} \mathrm{EW} \\
(\mathrm{m} \AA)\end{array}$ & $\begin{array}{c}\mathrm{D}_{1} \mathrm{EW} \\
(\mathrm{m} \AA)\end{array}$ & & $\begin{array}{c}\mathrm{D}_{2} \mathrm{EW} \\
(\mathrm{m} \AA)\end{array}$ & $\begin{array}{c}\mathrm{D}_{1} \mathrm{EW} \\
(\mathrm{m} \AA)\end{array}$ \\
\hline day -5 & $894 \pm 3$ & $649 \pm 3$ & & $653 \pm 2$ & $498 \pm 2$ \\
day 0 & $883 \pm 5$ & $661 \pm 5$ & & $655 \pm 3$ & $512 \pm 3$ \\
day +10 & $906 \pm 20$ & $695 \pm 20$ & & $670 \pm 12$ & $537 \pm 12$ \\
day +12 & $949 \pm 4$ & $702 \pm 5$ & & $711 \pm 3$ & $549 \pm 3$ \\
day +84 & $1006 \pm 7$ & $766 \pm 7$ & & $759 \pm 4$ & $603 \pm 4$ \\
\hline
\end{tabular}

It is worth noting that all of the $\mathrm{Na} \mathrm{D}$ absorption features, including the variable component, are blueshifted relative to the strongest absorption component at $5931.8 \AA(2130 \mathrm{~km}$ $\mathrm{s}^{-1}$ ), which we presume corresponds to the local interstellar medium (ISM) velocity (see Sections 3.6 and 3.7). In SN 2006X and SN 1999cl, the variable absorption was also blueshifted compared to the ISM absorption (Patat et al. 2007a; Blondin et al. 2009), perhaps providing a clue as to the origin of the material responsible for the varying absorption.

Having determined the EW of the variable absorption, we would now like to constrain the column density and line width of the absorbing gas. To isolate the changing component of the absorption profile, we examine the difference between each spectrum and the first one. These difference spectra are displayed in Figure 4. At day 0 (maximum light), we do not detect any obvious difference signal from 5 days earlier, despite the possible small increase in EW reported for the $\mathrm{D}_{1}$ line in Table 2 . Between day -5 and day +10 , however, a clear Gaussian line emerges in the difference spectrum (Figure 4, second row from the top). As expected from the EW measurements above, the strength of this line increases through day +84 , and we also find that the Doppler parameter of the line grows from $4.2 \pm$ $0.7 \mathrm{~km} \mathrm{~s}^{-1}$ in the (day +10 minus day -5 ) spectrum to $10.7 \pm 0.2 \mathrm{~km} \mathrm{~s}^{-1}$ in the (day +84 minus day -5 ) spectrum (see Table 3 ). The line in the (day +10 minus day -5 ) difference spectrum appears close to symmetric, but stronger hints of asymmetry in the later difference spectra suggest that some of this increase in line width is resulting from the addition of a second blended absorption component.

\subsection{Column Density of the Variable Component}

We see in Table 3 that the change in the EW of the $D_{2}$ line is very similar to the change in the $\mathrm{EW}$ of the $\mathrm{D}_{1}$ line at all epochs. At $1 \sigma$ significance, $0.78 \leqslant \Delta \mathrm{EW}_{\mathrm{D}_{1}} / \Delta \mathrm{EW}_{\mathrm{D}_{2}} \leqslant 1.03$ over the full set of difference spectra. Following Spitzer (1978, Equations (3)-(49) to (3)-(51)), we can use a curve-of-growth analysis to determine the observed EW for any combination of column density and line width. Using the derived Doppler parameters for the difference spectra given in Section 3.3, we calculate the change in EW that would be observed for a small increase in column density for all column densities between $10^{9}$ $\mathrm{cm}^{-2}$ and $10^{14.5} \mathrm{~cm}^{-2}$. For a given line width, these calculations demonstrate that increasing the equivalent width of the $D_{2}$ and $\mathrm{D}_{1}$ lines by the same amount is only possible for a narrow range of column densities, as illustrated in Figure 5. For $b=4.2 \mathrm{~km}$ $\mathrm{s}^{-1}$, we find that $12.0 \leqslant \log N_{\mathrm{Na}} \leqslant 12.3$, for $b=5.3 \mathrm{~km} \mathrm{~s}^{-1}$, we find that $12.1 \leqslant \log N_{\mathrm{Na}} \leqslant 12.4$, and for $b=10.7 \mathrm{~km} \mathrm{~s}^{-1}$, we find that $12.4 \leqslant \log N_{\mathrm{Na}} \leqslant 12.7$. We therefore conclude that the column density of the varying component of the absorption is $N_{\mathrm{Na} \text { I }} \approx 2.5 \times 10^{12} \mathrm{~cm}^{-2}$. Depending on the Doppler parameter, such a column density corresponds to a total $\mathrm{Na} \mathrm{D}_{2} \mathrm{EW}$ of

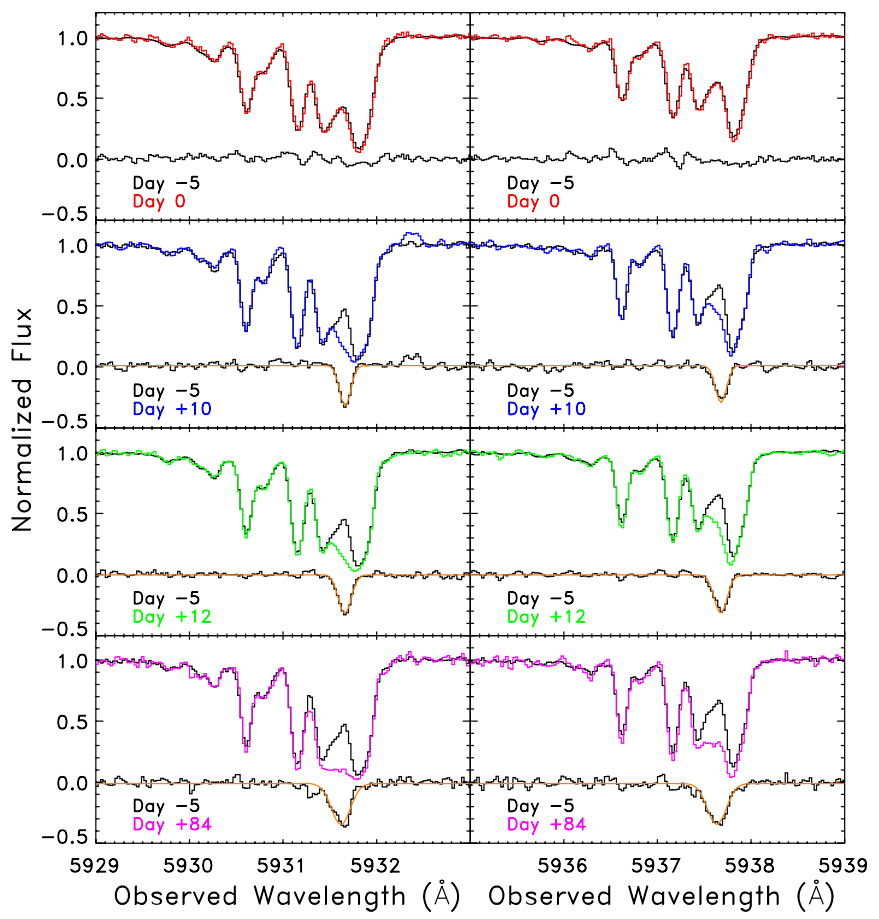

Figure 4. Differences in the $\mathrm{Na} \mathrm{D}$ lines between later epochs and the first spectrum. The left panels show the $\mathrm{D}_{2}$ lines and the right panels the $\mathrm{D}_{1}$ lines. The top curves in each panel are the observed spectra, and the black line at the bottom of each panel is the difference between the two spectra plotted in that panel. The orange curves are Gaussian fits to the difference spectra. There are no significant differences between the first two spectra (at and before maximum light), but the later spectra all show additional absorption at 5931.7 and $5937.7 \AA$.

200-300 m $\AA$ for this component, consistent with the results shown in Table 2.

It is evident from Figure 5 that the column densities indicated by the first two difference spectra (blue dashed and red dotted curves) are not consistent with the column density preferred by the third difference spectrum (gray dot-dashed curve). We suspect that the reason for this inconsistency is that the third difference spectrum contains multiple blended components, making the measured line width larger than the true line width as noted in Section 3.3. However, without a higher-resolution spectrum that separates the two components, we do not have conclusive evidence in favor of this interpretation.

\subsection{Calcium H\&K Absorption Lines at High Resolution}

The Keck/HIRES spectra extend far enough to the blue that we also detect $\mathrm{Ca}$ II $\mathrm{H} \& \mathrm{~K}$ absorption lines from both the Milky Way and the host galaxy, although the $\mathrm{S} / \mathrm{N}$ of the day +90 spectrum in the blue is too low to be useful. We compare the hostgalaxy absorption profile in $\mathrm{Ca} \mathrm{K}$ and $\mathrm{Na}_{2}$ in Figure 6, where a close correspondence between the absorption components in each species is visible.

Unlike the Na lines, though, we detect no statistically significant changes in the $\mathrm{Ca}$ absorption profile with time. Even nearly three months after maximum light, the profile shape (Figure 7) and total EW (Table 4) match those from before maximum light within the uncertainties. However, while changes in the $\mathrm{Ca} \mathrm{EW}$ as large as those seen in the $\mathrm{Na}$ lines can be ruled out, the low $\mathrm{S} / \mathrm{N}$ of the late-time spectra prevents us from being able to place strong constraints on smaller variations. 
Table 3

Na D Difference Spectra Fits

\begin{tabular}{ccccc}
\hline \hline Epochs & $\begin{array}{c}\mathrm{D}_{2} \text { EW Change } \\
(\mathrm{m \AA})\end{array}$ & $\begin{array}{c}\mathrm{D}_{1} \text { EW Change } \\
(\mathrm{m \AA})\end{array}$ & $\begin{array}{c}\mathrm{D}_{2} \text { Velocity } \\
\left(\mathrm{km} \mathrm{s}^{-1}\right)\end{array}$ & $\begin{array}{c}\mathrm{D}_{2} \text { Doppler Parameter } \\
\left(\mathrm{km} \mathrm{s}^{-1}\right)\end{array}$ \\
\hline day $+10-$ day -5 & $50 \pm 4$ & $45 \pm 5$ & $2123.1 \pm 0.2$ & $4.2 \pm 0.7$ \\
day $+12-$ day -5 & $55 \pm 1$ & $54 \pm 2$ & $2122.9 \pm 0.1$ & $5.3 \pm 0.2$ \\
day +84 - day -5 & $85 \pm 2$ & $85 \pm 2$ & $2120.8 \pm 0.1$ & $10.7 \pm 0.2$ \\
\hline
\end{tabular}

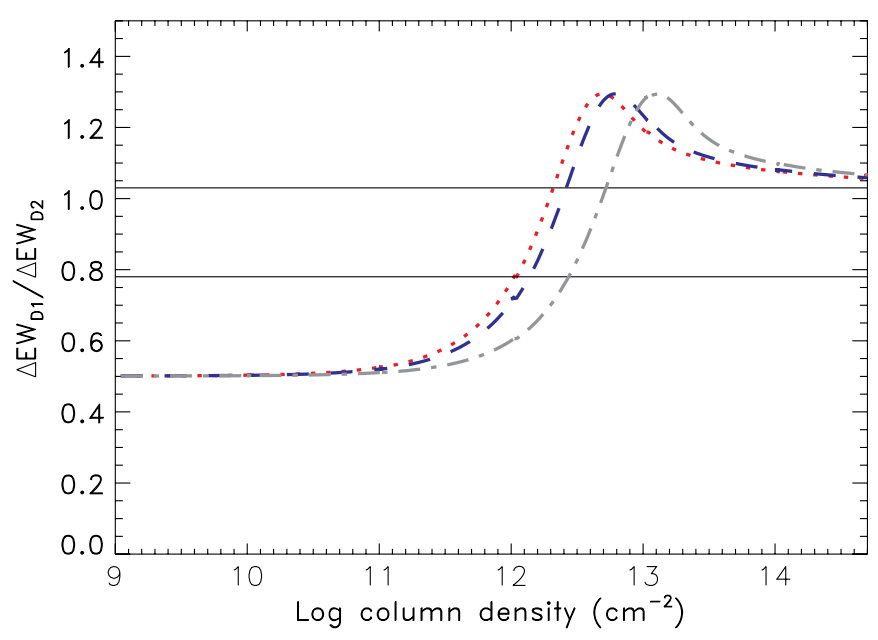

Figure 5. Ratio of the change in the EW of the $\mathrm{Na}$ I $\mathrm{D}_{1}$ line to that of the $\mathrm{D}_{2}$ line for a small increase in the absorbing column as a function of column density. The dotted red curve represents the EW ratio for a Doppler parameter of $b=4.2 \mathrm{~km} \mathrm{~s}^{-1}$, appropriate for the (day +10 minus day -5 ) spectrum, the dashed blue curve for a Doppler parameter of $b=5.3 \mathrm{~km} \mathrm{~s}^{-1}$, appropriate for the (day +12 minus day -5 ) spectrum, and the dot-dashed gray curve for a Doppler parameter of $b=10.7 \mathrm{~km} \mathrm{~s}^{-1}$, appropriate for the (day +84 minus day $-5)$ spectrum. The horizontal solid lines delineate the $1 \sigma$ constraints placed by the observed $\mathrm{EW}$ ratios, $0.78 \leqslant \Delta \mathrm{EW}_{\mathrm{D}_{1}} / \Delta \mathrm{EW}_{\mathrm{D}_{2}} \leqslant 1.03$. The observed ratio of the EW changes can only be obtained over a narrow range of column densities from $10^{12} \mathrm{~cm}^{-2}$ to $10^{12.7} \mathrm{~cm}^{-2}$.

(A color version of this figure is available in the online journal.)

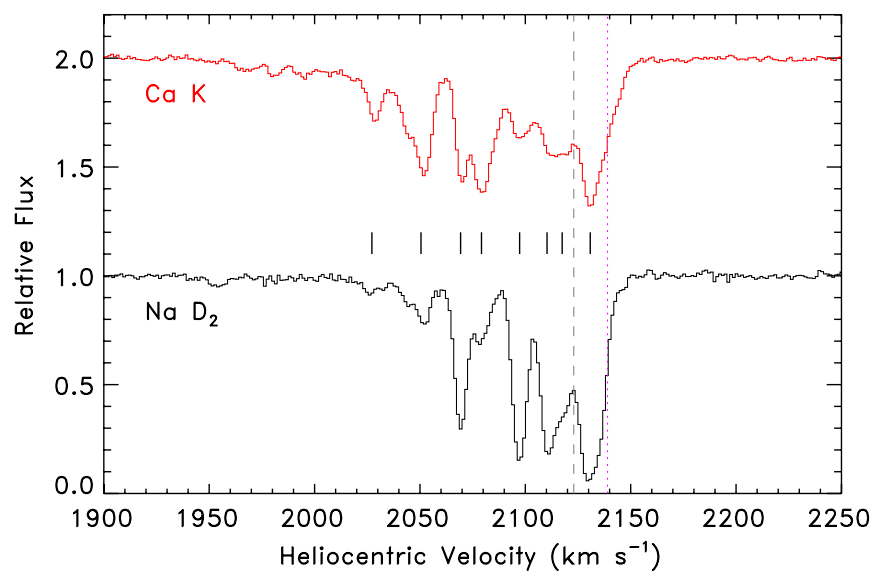

Figure 6. Comparison between the host-galaxy absorption profile in the $\mathrm{Ca}$ II $\mathrm{K}$ (upper spectrum, in red) and $\mathrm{Na}$ I $\mathrm{D}_{2}$ (lower spectrum, in black) lines, both from five days before maximum light. The vertical dashed gray line is at the central velocity of the variable $\mathrm{Na}$ absorption component. Each $\mathrm{Na}$ absorption component, including the variable one, has a visible $\mathrm{Ca}$ counterpart (and vice versa), with the possible exception of some very weak blueshifted features between 1950 and $2000 \mathrm{~km} \mathrm{~s}^{-1}$. The relative strengths of the $\mathrm{Na}$ and $\mathrm{Ca}$ lines, however, vary significantly from one component to the next. The $\mathrm{Ca}$ lines also appear to have much more prominent wings than the $\mathrm{Na}$ absorption. The vertical ticks mark the velocities of individual absorption components inferred from Gaussian fits to the Na lines, and the vertical dotted magenta line indicates the velocity of the $\mathrm{H} \alpha$ emission line discussed in Section 3.7.

(A color version of this figure is available in the online journal.)
Table 4

Ca H \& K Equivalent Widths

\begin{tabular}{|c|c|c|c|c|}
\hline \multirow[b]{2}{*}{ Epoch } & \multicolumn{2}{|c|}{ Full Profile } & \multicolumn{2}{|c|}{ Red Side } \\
\hline & $\begin{array}{c}\text { K EW } \\
(\mathrm{m} \AA)\end{array}$ & $\begin{array}{c}\text { H EW } \\
(\mathrm{m} \AA)\end{array}$ & $\begin{array}{c}\text { K EW } \\
(\mathrm{m} \AA)\end{array}$ & $\begin{array}{c}\text { H EW } \\
(\mathrm{m} \AA)\end{array}$ \\
\hline day -5 & $653 \pm 2$ & $382 \pm 2$ & $301 \pm 1$ & $174 \pm 1$ \\
\hline day 0 & $677 \pm 9$ & $397 \pm 9$ & $308 \pm 4$ & $178 \pm 5$ \\
\hline day +12 & $629 \pm 6$ & $388 \pm 6$ & $294 \pm 3$ & $186 \pm 3$ \\
\hline day +84 & $749 \pm 32$ & $413 \pm 28$ & $343 \pm 16$ & $153 \pm 15$ \\
\hline
\end{tabular}

3.6. Other Absorption Features

In addition to the $\mathrm{Ca} \mathrm{H} \& \mathrm{~K}$ and $\mathrm{Na} \mathrm{D}$ lines, we also searched the spectra for the additional absorption features identified by Patat et al. (2007a) and Cox \& Patat (2008) in SN 2006X. In the highest $\mathrm{S} / \mathrm{N}$ (day -5) spectrum, we detect the $\mathrm{CH}^{+}$ $3957.70 \AA$ and $4232.55 \AA$ lines at a velocity of $2130 \mathrm{~km} \mathrm{~s}^{-1}$, exactly matching the velocity of the strongest $\mathrm{Na} \mathrm{D}$ absorption. Diffuse interstellar bands are also visible at $5780 \AA$ and $6283 \AA$ (rest wavelengths). We do not detect Ca I at $4226.73 \AA$. $\mathrm{CH}$ at $4300.30 \AA$, the CN vibrational band, or K I at $7699 \AA$.

\subsection{Ha Emission}

All six of our high-resolution spectra cover the expected wavelength of the redshifted $\mathrm{H} \alpha$ line. However, the fiber spectrum from the HET is not suitable for investigating possible $\mathrm{H} \alpha$ emission from the SN because of the lack of local sky subtraction, so we only consider the five high-resolution Keck spectra in this section.

We detect a narrow $\mathrm{H} \alpha$ emission line at the position of the SN at all epochs of our observations. The HIRES spectra were obtained with a slit $7^{\prime \prime}$ long and either 0 '.86 or 1'.15 wide, and faint $\mathrm{H} \alpha$ emission from the host galaxy is visible all the way along the slit. However, careful sky subtraction reveals that there is also additional emission that is spatially unresolved and coincident with the position of the SN (see Figures 8 and 9). This line is also at a slightly different velocity than the extended host-galaxy emission.

From the HIRES data, we measure the EW, velocity, and line width of the $\mathrm{H} \alpha$ emission at the position of the $\mathrm{SN}$; the results are listed in Table 5. At $v=2139 \mathrm{~km} \mathrm{~s}^{-1}$, the $\mathrm{H} \alpha$ emission is offset by $16 \mathrm{~km} \mathrm{~s}^{-1}$ from the velocity of the variable sodium line (and has a higher velocity than any of the main sodium components), but is similar to the host-galaxy rotation velocity at the radius of the SN measured from a long-slit spectrum by Afanasyev et al. (1992). We then use the observed light curve and a comprehensive set of low-resolution spectra of SN 2007le from the CfA Supernova Archive to derive absolute flux calibrations for the spectra. In order to remove contaminating host-galaxy light (since the CfA spectra were obtained with a wide slit), the spectra are first scaled to the observed $V$-band magnitudes of the $\mathrm{SN}$ and then warped to match the $B$ magnitudes as well. From these flux-calibrated spectra, we determine the SN continuum level at the position of $\mathrm{H} \alpha(6610 \AA)$, scaling the spectra up 

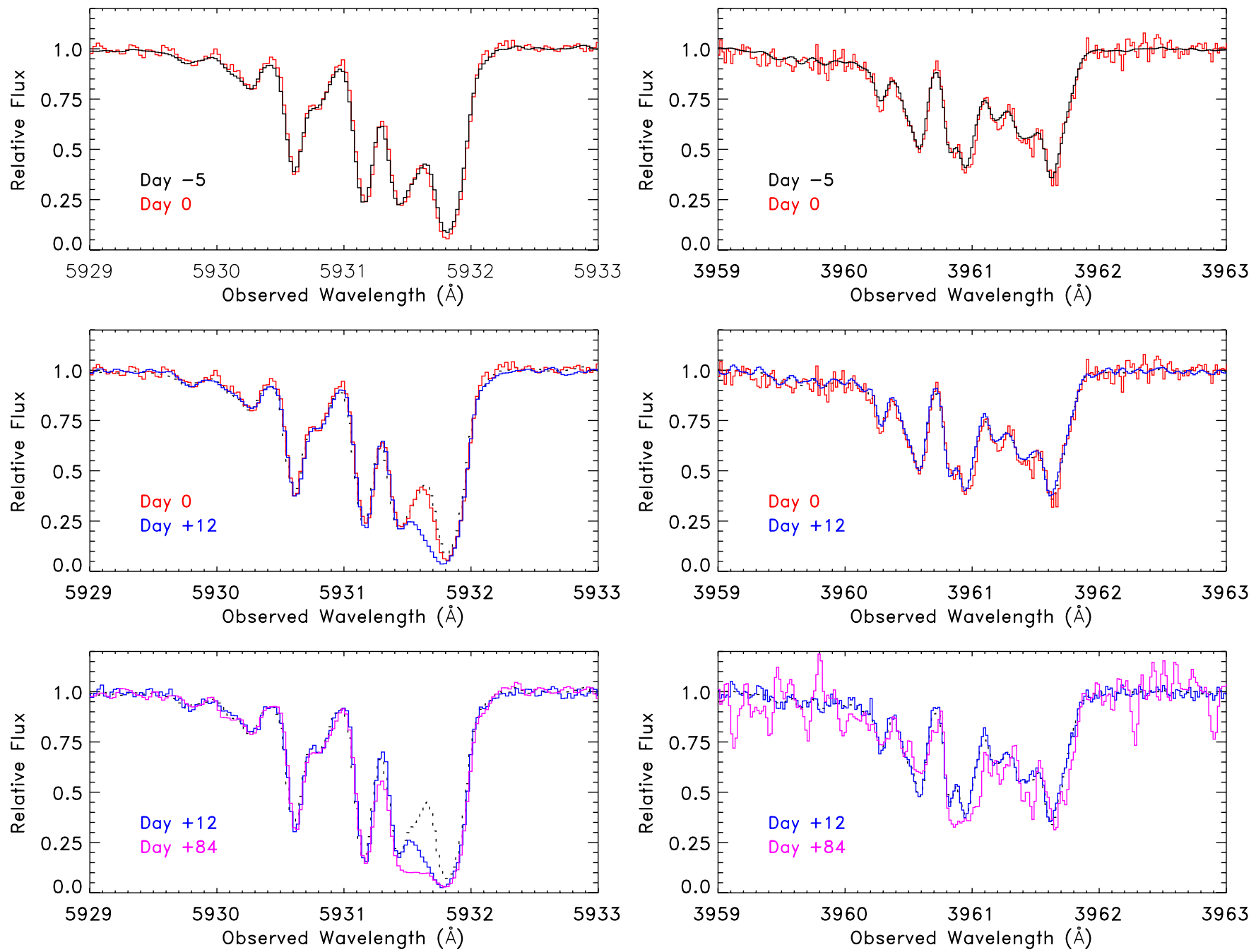

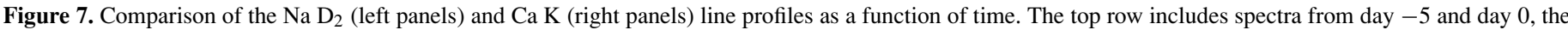

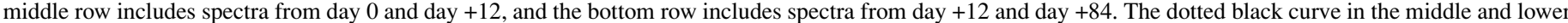
panels represents the day -5 spectrum. Over the time period when significant changes are visible in the Na lines there are no detectable variations in the Ca lines.

Table 5

Emission-Line Widths and Fluxes

\begin{tabular}{|c|c|c|c|c|c|c|c|c|}
\hline Epoch & $\begin{array}{c}\mathrm{H} \alpha \mathrm{EW} \\
(\mathrm{m} \AA)\end{array}$ & $\begin{array}{c}\mathrm{H} \alpha \text { Velocity } \\
\left(\mathrm{km} \mathrm{s}^{-1}\right)\end{array}$ & $\begin{array}{c}\text { H } \alpha \text { Line Width } \\
\left(\mathrm{km} \mathrm{s}^{-1}\right)\end{array}$ & $\begin{array}{c}\mathrm{H} \alpha \text { Flux } \\
\left(\mathrm{erg} \mathrm{cm}^{-2} \mathrm{~s}^{-1}\right)\end{array}$ & $\begin{array}{c}\mathrm{H} \beta \text { Flux } \\
\left(\mathrm{erg} \mathrm{cm}^{-2} \mathrm{~s}^{-1}\right)\end{array}$ & $\begin{array}{c}{[\mathrm{O} \text { III }] \text { Flux }} \\
\left(\mathrm{erg} \mathrm{cm}^{-2} \mathrm{~s}^{-1}\right) \\
\end{array}$ & $\begin{array}{c}{[\mathrm{N} \text { II }] \text { Flux }^{\mathrm{a}}} \\
\left(\mathrm{erg} \mathrm{cm}^{-2} \mathrm{~s}^{-1}\right) \\
\end{array}$ & $\begin{array}{c}\text { [S II }] \text { Flux }^{\mathrm{b}} \\
\left(\mathrm{erg} \mathrm{cm}^{-2} \mathrm{~s}^{-1}\right) \\
\end{array}$ \\
\hline day -5 & $22 \pm 3$ & $2137.8 \pm 1.1$ & $25.9 \pm 4.5$ & $(1.5 \pm 0.2) \times 10^{-16}$ & $\ldots{ }^{c}$ & $<5.8 \times 10^{-17}$ & $\ldots$ & $\ldots$ \\
\hline day 0 & $14 \pm 4$ & $2140.1 \pm 1.7$ & $17.9 \pm 4.0$ & $(1.2 \pm 0.3) \times 10^{-16}$ & $\ldots$ & $\ldots$ & $\ldots$ & $\ldots$ \\
\hline day +12 & $28 \pm 5$ & $2136.4 \pm 1.4$ & $25.4 \pm 3.3$ & $(1.5 \pm 0.3) \times 10^{-16}$ & $\ldots$ & $\ldots$ & $\ldots$ & $\ldots$ \\
\hline day +84 & $288 \pm 9$ & $2139.3 \pm 0.3$ & $30.8 \pm 0.7$ & $(2.1 \pm 0.1) \times 10^{-16}$ & $3.9 \pm 0.7 \times 10^{-17}$ & $<1.5 \times 10^{-17}$ & $(6.0 \pm 0.7) \times 10^{-17}$ & $(2.7 \pm 0.5) \times 10^{-17}$ \\
\hline day +90 & $301 \pm 41$ & $2139.5 \pm 1.0$ & $24.5 \pm 2.6$ & $(1.8 \pm 0.3) \times 10^{-16}$ & $\ldots$ & $\ldots$ & $\ldots$ & $\cdots$ \\
\hline
\end{tabular}

Notes.

a The listed value is for the stronger $[\mathrm{N} \mathrm{II}] \lambda 6583$ line.

$\mathrm{b}$ The listed value is for the stronger $[\mathrm{S}$ II] $\lambda 6717$ line.

${ }^{c}$ We have listed upper limits only where the data provide useful constraints; [O III] upper limits are at $4 \sigma$ significance.

and down as necessary to account for the change in the SN's $R$ magnitude between the time of observation for each highresolution spectrum and the nearest low-resolution spectrum (the time differences are less than two days for days -5 and +12 , six days for day 0 , and five and eleven days for days +84 and +90 , respectively). We then convert the high-resolution EWs to fluxes in absolute units with the measured continuum levels.

In principle, the $\mathrm{H} \alpha$ emission could originate either from an $\mathrm{H}$ II region projected within $140 \mathrm{pc}$ (corresponding to the $\approx 1^{\prime \prime}$ slit width) of the explosion or from the SN itself. In the case of an $\mathrm{H}$ II region, one would expect other lines such as [O III] $\lambda \lambda 4959,5007,[\mathrm{~N}$ II] $\lambda \lambda 6548,6583$, and [S II] $\lambda \lambda 6717,6731$ to be visible depending on the $S / N$. In the day +84 spectrum, which is the best for this purpose because of its relatively high $\mathrm{S} / \mathrm{N}$ at red wavelengths and the faint magnitude of the $\mathrm{SN}$ at late times, we indeed detect $\mathrm{H} \beta$ and both lines of the [N II] and [S II] doublets. [O III], though, which is generally the brightest of the forbidden metal lines in $\mathrm{H}$ II regions (and comparable to 

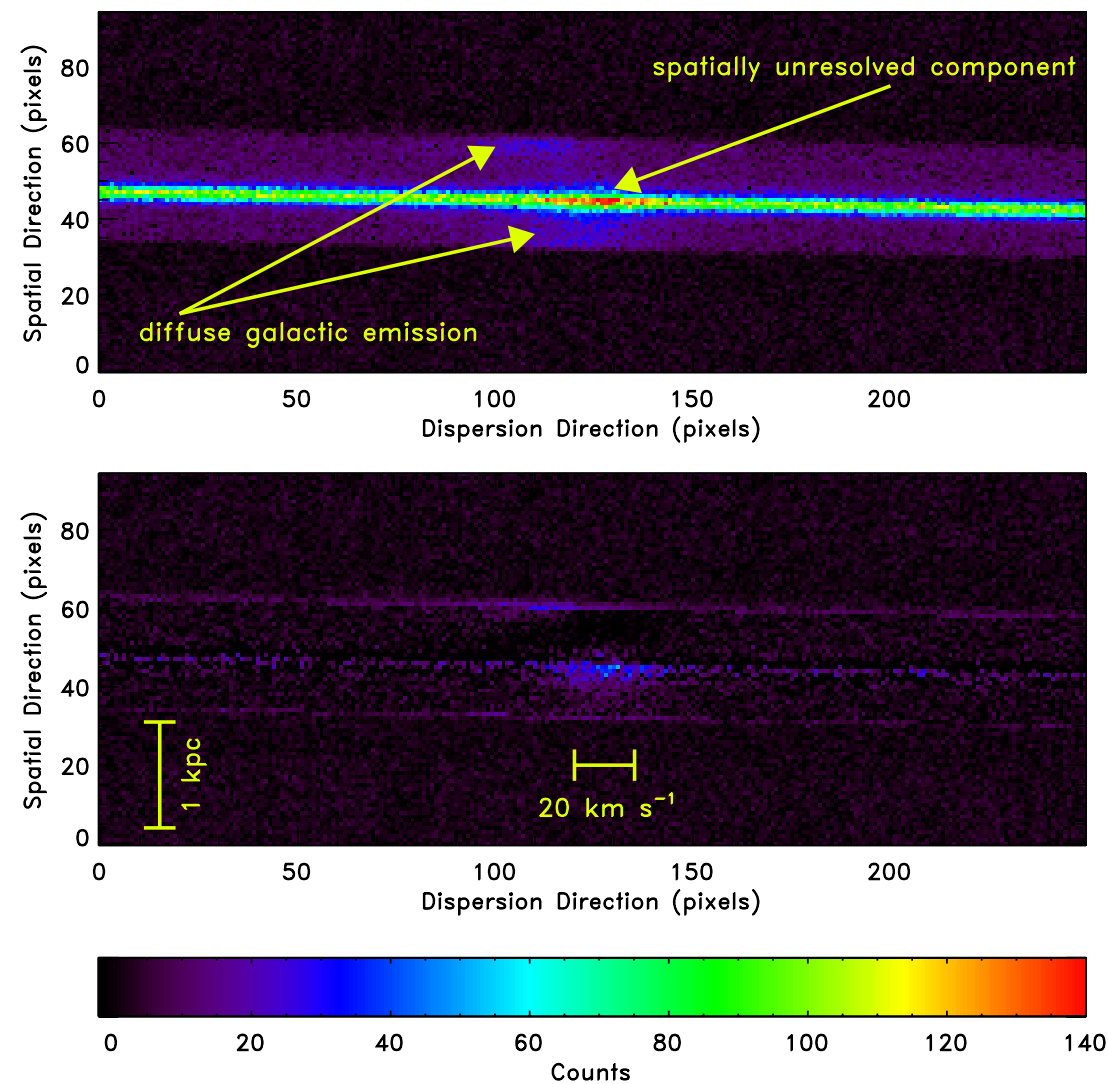

Figure 8. Two-dimensional spectra of SN 2007le around the redshifted $\mathrm{H} \alpha$ line. These data are from day +84 , where the $\mathrm{S} / \mathrm{N}$ of the $\mathrm{H} \alpha$ detection is highest. The top panel shows a portion of the echelle order containing $\mathrm{H} \alpha$ in the coadded raw frames (the only processing was to remove cosmic rays). Diffuse $\mathrm{H} \alpha$ emission is visible across the slit, with a velocity that changes from one side to the other, but an additional distinct component is present right on top of the SN continuum. In the lower panel, the continuum emission and the average sky light across the slit have been subtracted. The sky fitting is not perfect, since the diffuse H $\alpha$ was not symmetrically distributed around the supernova, but it is clear that there is excess $\mathrm{H} \alpha$ light at the position of the SN compared with the surrounding areas of the host galaxy. Fully reduced and extracted one-dimensional spectra of this emission are displayed in Figure 9.

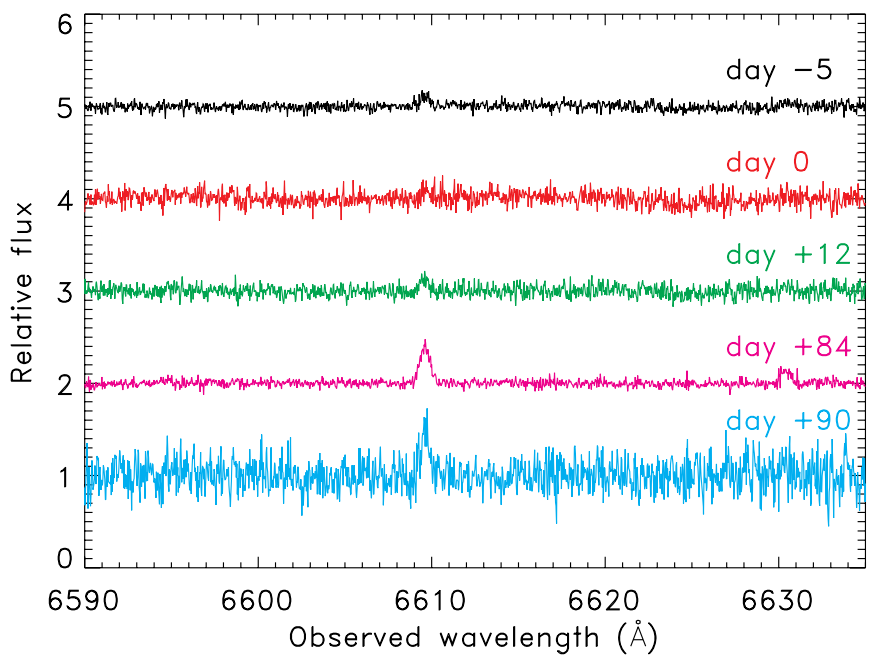

Figure 9. High-resolution spectra of SN 2007le around the redshifted $\mathrm{H} \alpha$ and $[\mathrm{N}$ II] lines. From top to bottom, the spectra are from days $-5,0,+12,+84$, and +90 (the day +10 spectrum is excluded because local sky subtraction cannot be done on a fiber spectrum). A constant offset has been added to the top four spectra for clarity, and the days $-5,0$, and +12 spectra have been multiplied by a factor of 3 before adding the offset to make the emission line visible on this display scale. The emission line appears to be stronger at late times primarily because the supernova continuum on top of which it is superimposed has faded by more than an order of magnitude.

or brighter than $\mathrm{H} \beta$ ), remains undetected at all epochs. [O III] fluxes more than an order of magnitude weaker than $\mathrm{H} \alpha$ are uncommon in H II regions (e.g., Kennicutt et al. 2003; Magrini et al. 2007).

On the other hand, if the $\mathrm{H} \alpha$ emission is related to the $\mathrm{SN}$, changes in the $\mathrm{H} \alpha$ flux with time would seem likely (although since such emission has never been detected, its exact characteristics are unknown). From the day -5 spectrum through the day +12 spectrum (spanning about 2.5 weeks), we detect no variability in the flux. In the day +84 spectrum more than two months later, however, the $\mathrm{H} \alpha$ flux is $\sim 40 \%$ higher than at day +12 . If the comparison is made only between days +12 and +84 , the significance of this change is just $2 \sigma$, but if we combine the measurements from days $-5,0$, and +12 together then the day +84 increase is significant at the $4 \sigma$ level. The low-S/N day +90 spectrum is consistent with both the early time and day +84 measurements. Taken as a whole, we consider these results to be a tentative indication of $\mathrm{H} \alpha$ emission from SN 2007le. More conclusive evidence of the nature of the $\mathrm{H} \alpha$ emission awaits late-time imaging and spectroscopy to determine whether or not there is an $\mathrm{H}$ II region coincident with the position of the SN.

If the $\mathrm{H} \alpha$ emission is associated with the $\mathrm{SN}$, its luminosity is related to the amount of gas present. The observed $\mathrm{H} \alpha$ emission has a luminosity of $1.6 \times 10^{35} \mathrm{erg} \mathrm{s}^{-1}$ (for the average $\mathrm{H} \alpha$ flux of $1.5 \times 10^{-16} \mathrm{erg} \mathrm{cm}^{-2} \mathrm{~s}^{-1}$ ). If we follow the calculations of Leonard (2007), such a luminosity corresponds to $\sim 0.025 M_{\odot}$ of solar-abundance material in the circumstellar environment, in reasonable agreement with theoretical predictions (Marietta et al. 2000; Mattila et al. 2005; Pakmor et al. 2008). However, 
those computations were specifically aimed at modeling $\mathrm{H} \alpha$ emission at very late times $($ day +380$)$ and may not apply to the much earlier epochs at which we observed SN 2007le. Using the simple model presented by Patat et al. (2007a) as an alternative suggests a hydrogen mass of $\sim 0.001 M_{\odot}$ for the observed $\mathrm{H} \alpha$ luminosity. The presence of hydrogen in the CSM has also been indirectly inferred from the broadening of absorption features in the earliest spectra of SNe Ia (Mazzali et al. 2005a). In this scenario, $\sim 0.005 M_{\odot}$ of material with a solar composition is necessary to provide the electron density that is required to trap photons and cause absorption in lines such as $\mathrm{Ca} \mathrm{H} \& \mathrm{~K}$ or Si II $\lambda 6355$ (Mazzali et al. 2005b; Tanaka et al. 2008).

\section{DISCUSSION}

For SN 2006X, Patat et al. (2007a) suggested a model in which the progenitor system consisted of a white dwarf and a red giant, with either the stellar wind from the red giant or repeated nova eruptions blowing off several possibly asymmetric shells of material before the explosion. These circumstellar shells were proposed to be the location of the material responsible for the variable $\mathrm{Na} \mathrm{D}$ absorption. Given timescales of the order of decades for the mass loss and the observed velocities of the variable lines, the absorbing material would be $\sim 10^{16} \mathrm{~cm}$ away from the star at the time of explosion. This scenario envisions that the $\mathrm{Na}$ I atoms (with an ionization potential of $5.1 \mathrm{eV}$ ) are ionized by the UV radiation from the SN, and then slowly recombine over the following weeks to produce strengthening $\mathrm{Na} \mathrm{D}$ absorption lines. The absence of variations in the $\mathrm{Ca} \mathrm{H} \&$ $\mathrm{K}$ lines is then attributed to the much higher ionization potential of $\mathrm{Ca}$ II $(11.9 \mathrm{eV})$, which prevents most of the $\mathrm{Ca}$ ions from being affected by the $\mathrm{SN}$ radiation field.

However, Chugai (2008) has challenged this interpretation, arguing that the physical conditions expected in a red giant wind are not compatible with strong $\mathrm{Na} \mathrm{D}$ absorption. ${ }^{25}$ In light of these calculations, plus the confirmation from SN 2007le and SN 1999cl (Blondin et al. 2009) that the variable Na absorption phenomenon is not unique to SN 2006X, we now reconsider the nature of the absorbing material.

\subsection{Photoionization and Recombination Timescales}

If the changes in the $\mathrm{Na} \mathrm{D}$ absorption lines are a result of recombination, then the spectra tell us directly that the recombination timescale is $\sim 10$ days (e.g., Figure 3 ). Since

$$
\tau_{\text {recomb }}=\frac{1}{\alpha n_{e}}
$$

where $\alpha$, the Na I recombination coefficient, is $1.43 \times 10^{-13} \mathrm{~cm}^{3}$ $\mathrm{s}^{-1}$ at $T=10^{4} \mathrm{~K}$ (Badnell 2006; see Section 4.2 for a justification of this temperature range), we find that the electron density must be $n_{e} \approx 8 \times 10^{6} \mathrm{~cm}^{-3}$. (For $T=2 \times 10^{3} \mathrm{~K}$, $\alpha=6.18 \times 10^{-13} \mathrm{~cm}^{3} \mathrm{~s}^{-1}$, and the required electron density will be lower by a corresponding factor.) We note that such a high electron density necessitates that hydrogen must be partially or mostly ionized; no other plausible source could provide so many electrons (as mentioned in Section 3.7, Mazzali et al. 2005a suggest that electrons provided by hydrogen can also explain the origin of high-velocity features, as were present in

\footnotetext{
25 Note, however, that the underprediction of $\mathrm{Na} \mathrm{I}$ is a known issue in ionization models (e.g., Mazzali et al. 1997). The reason for this problem is not understood, but it could provide an explanation for the observed EW ratio between $\mathrm{Na}$ I and $\mathrm{Ca}$ II exceeding the expected one by a large factor (see Section 4.2)
}

SN 2007le, at early times). Even with full ionization, though, the physical density required in the absorbing material is quite high.

In order for a significant fraction of neutral sodium to be present, the photoionization rate should be comparable to or slower than the recombination rate, or alternatively, the recombination time should be shorter than the photoionization time. Following Murray et al. (2007), we write

$$
\tau_{\text {ion }}=\frac{4 \pi r^{2}\langle h \nu\rangle}{a_{\mathrm{Na} I} L_{\mathrm{UV}}},
$$

where $r$ is the distance between the $\mathrm{SN}$ and the absorbing material, $a_{\mathrm{Na}} \approx 10^{-19} \mathrm{~cm}^{2}$ is the photoionization cross section (Verner et al. 1996), $L_{\mathrm{UV}}$ is the UV luminosity of the $\mathrm{SN}$, and $\langle h v\rangle$ corresponds to the photon energy required to ionize a $\mathrm{Na}$ I atom. We do not have UV photometry of SN 2007le, but to the degree that UV light curves of SNe Ia are fairly homogeneous (Brown et al. 2009), it is reasonable to substitute another similar SN. We therefore use the Swift light curve of the well-observed normal SN Ia 2007af from Brown et al. to estimate the UV luminosity. SN 2007af reached a peak Vega magnitude of $\sim 16.2$ in the Swift $U V W 2$ filter a few days before the $B$-band maximum. The $U V W 2$ filter has a central wavelength of $1928 \AA$, a full width at half-maximum intensity (FWHM) of $657 \AA$, and a photometric zero point of $17.35 \pm$ $0.03 \mathrm{mag}$ (Poole et al. 2008). We use the distance modulus of SN 2007af, $32.06 \mathrm{mag}$ (Simon et al. 2007), to deduce an absolute magnitude of $M_{U V W 2}=-15.86$, which suggests an apparent peak magnitude for SN 2007le of 16.49. With the zero point of the $U V W 2$ filter, this magnitude translates to a Swift count rate of 2.2 counts $\mathrm{s}^{-1}$. Poole et al. (2008) provide a conversion between the count rate and the flux density that depends only slightly on the source spectrum $\left(\sim 6.1 \times 10^{-16} \mathrm{erg} \mathrm{cm}^{-2}\right.$ $\mathrm{s}^{-1} \AA^{-1}$ ), so integrating over the filter bandwidth yields a flux of $8.8 \times 10^{-13} \mathrm{erg} \mathrm{cm}^{-2} \mathrm{~s}$. We therefore estimate a $U V W 2$ luminosity for SN 2007le of $9 \times 10^{40} \mathrm{erg} \mathrm{s}^{-1}$. Note that this bandpass corresponds reasonably closely to the wavelengths that can ionize $\mathrm{Na}$ I atoms most efficiently $(\lambda<2412 \AA)$. Using Equation (2), we can now calculate that the ionization timescale is

$$
\tau_{\text {ion }}=1.1\left(\frac{r}{10^{16} \mathrm{~cm}}\right)^{2} \mathrm{~s} .
$$

For $r \lesssim 3$ pc, then, $\tau_{\text {ion }} \ll \tau_{\text {recomb }}$, and this estimate suggests that the $\mathrm{Na}$ should be essentially fully ionized around maximum light.

Although the UV luminosity of the SN declines with time, the Swift data show that the decline is rather slow: $\sim 1$ mag in 10 days (Brown et al. 2009). Even several weeks after peak, when the high-resolution spectroscopy demonstrates that significant changes in the $\mathrm{Na} \mathrm{D}$ line profile have already occurred, Equations (1) and (3) indicate that recombination (at the assumed densities) should only be occurring at relatively large radii, calling into question the hypothesis that circumstellar material is responsible for the varying $\mathrm{Na}$ absorption. These simple calculations are in qualitative agreement with the results of Chugai (2008) and indicate that more sophisticated modeling is required.

\subsection{Photoionization Modeling}

Because the simple ionization and recombination timescale arguments do not provide obvious answers as to why the $\mathrm{Na}$ I column density is changing, we now consider more detailed 
photoionization models of the SN environment. Starting with a synthetic UV spectrum for a SN Ia from Nugent et al. (1995) as the ionizing source, we carry out calculations with the photoionization code Cloudy (Ferland et al. 1998, ver. 07.02). We use the UV spectra of SN 2001eh and SN 2001ep (Sauer et al. 2008) to normalize the total number of UV-IR photons (1500-12000 ̊) in the model spectrum. ${ }^{26}$ The model spectrum is only available for the time of maximum brightness, so we assume that the light curve at all UV wavelengths follows the SN 2007af $U V W 2$ light curve from Brown et al. (2009). (We have to combine observations of multiple SNe here because there are few nearby SNe Ia with both good UV light curves and UV spectroscopy.) This assumption means that the calculations are not explicitly time dependent, but we do not expect non-equilibrium effects to produce qualitative changes in the results discussed below. We also note that, while at longer UV wavelengths the model can be compared to observed spectra for validation, at the critical far-UV wavelengths that ionize hydrogen no such comparison with real data is possible. Borkowski et al. (2009) have recently carried out very similar calculations to investigate the possibility of detecting CSM interactions in spectra obtained before maximum light.

Guided by the hypothesis that recombination is responsible for the varying $\mathrm{Na}$ I column density, a few iterations of the model demonstrate that very large hydrogen densities are needed to produce significant $\mathrm{Na}$ recombination (for a solar abundance ratio). We therefore assume a hydrogen density of $n=2.4 \times 10^{7} \mathrm{~cm}^{-3}$ (somewhat higher than the electron density inferred in Section 4.1). We then calculate the $\mathrm{Na}$ I, Ca II, and $\mathrm{H}$ II fractions as a function of time and distance away from the SN. The results are illustrated in Figure 10. For distances greater than $\sim 0.1 \mathrm{pc}$ the fraction of $\mathrm{Ca}$ II ions is essentially constant with time, while the $\mathrm{Na}$ I fraction increases by approximately an order of magnitude over a time span of 30 days. Closer to the $\mathrm{SN}$, the Ca becomes more highly ionized (and the $\mathrm{Ca}$ II fraction varies with time) and the amount of neutral $\mathrm{Na}$ is negligible, supporting the idea that the source of the absorption must be located at distances larger than $10^{16} \mathrm{~cm}$.

Over the range of parameter space where these calculations indicate that the $\mathrm{Na}$ I column density will vary and the $\mathrm{Ca}$ II column density will not, the $\mathrm{EW}$ of the $\mathrm{Na}_{2}$ line is predicted to be less than or equal to the $\mathrm{EW}$ of the $\mathrm{Ca} \mathrm{K}$ line. At distances of less than $1 \mathrm{pc}$, the $\mathrm{Ca}$ absorption should be $\gtrsim 100$ times as strong as the $\mathrm{Na}$ absorption. Yet, we observe that $\mathrm{EW}_{\mathrm{NaD}}$ is larger than $\mathrm{EW}_{\mathrm{CaH} \& \mathrm{~K}}$ at all epochs (see Tables 2 and 4 and Figure 7). If the model described above is correct, we therefore require that nearly all of the $\mathrm{Ca}$ atoms in the CSM are locked up in dust grains, as is also typical in dense interstellar clouds (e.g., Spitzer 1954; Howard et al. 1963; Herbig 1968; Morton 1975). In the immediate vicinity of a SN, the destruction of dust grains is obviously a possibility. However, given sublimation temperatures of $\sim 1500 \mathrm{~K}$ for silicate and carbonaceous grains (Draine \& Salpeter 1979), calculations of grain temperatures with Cloudy using the dust grain model of van Hoof et al. (2004) indicate that dust can survive at distances greater than $0.05 \mathrm{pc}$ (unshaded region in Figure 10) over the relevant time period.

\footnotetext{
26 The normalization $N_{\mathrm{SN}}$ is chosen such that

$4 \pi d_{\mathrm{SN}}^{2} \int \lambda J_{\lambda}^{\mathrm{SN}} d \lambda=N_{\mathrm{SN}} \int \lambda J_{\lambda}^{\text {model }} d \lambda$, where $J_{\lambda}^{\text {model }}$ is the model spectrum, and $J_{\lambda}^{\mathrm{SN}}$ and $d_{\mathrm{SN}}$ are the $\mathrm{SN}$ spectrum and distance. The normalization factors for SN 2001ep and SN 2001eh agree to within 30\%, so we use $N_{\mathrm{SN}}=0.5\left(N_{\mathrm{ep}}+N_{\mathrm{eh}}\right)$.
}

\subsection{Physical Conditions in the Absorbing Gas}

The results presented in Section 4.2 point to distances of $\sim 0.1 \mathrm{pc}$ for the absorbing material. Figure 10 demonstrates that the EW of the $\mathrm{Ca}$ II absorption would remain constant for distances as small as $\sim 0.02 \mathrm{pc}$, but the fraction of neutral sodium so close to the $\mathrm{SN}$ is negligible. Distances greater than $1 \mathrm{pc}$ are also allowed by the photoionization modeling, but then the ionization fraction is very low (as a result of the weak UV field), requiring extremely high physical densities to produce large numbers of electrons. Of course, at such distances the galactic UV field may contribute significantly to the photoionization rate, rendering our calculations no longer applicable. At $d=$ $0.1 \mathrm{pc}$, the gas temperature is $\sim 5000 \mathrm{~K}$, and densities of $n_{\mathrm{H}} \approx 2.4 \times 10^{7} \mathrm{~cm}^{-3}$ are required to produce significant $\mathrm{Na}$ recombination. With such a high density, substantial amounts of dust could be present, and strong depletion of $\mathrm{Ca}$ atoms onto dust grains would explain the large observed ratio of $\mathrm{Na}$ I to $\mathrm{Ca}$ II. Given the derived density and a solar $\mathrm{Na}$ abundance of $12+\log (\mathrm{Na} / \mathrm{H})=6.17$ (Asplund et al. 2005), the total density of $\mathrm{Na}$ atoms is $n_{\mathrm{Na}}=35 \mathrm{~cm}^{-3}$. The $\mathrm{Na}$ I fraction under these conditions is $\sim 5 \times 10^{-4}$, yielding $n_{\mathrm{NaI}}=1.8 \times 10^{-2} \mathrm{~cm}^{-3}$. In order to obtain a column density of $\sim 2.5 \times 10^{12} \mathrm{~cm}^{-2}$, as derived in Section 3.4, the path length through the absorbing cloud must be $\sim 1.4 \times 10^{14} \mathrm{~cm}$. If such a clump of material were roughly spherical its mass could be quite small $\left(\sim 2 \times 10^{-7} M_{\odot}\right)$, but a clump of radius $10^{14} \mathrm{~cm}$ at a distance of $10^{17} \mathrm{~cm}$ would only occult a small fraction of the $\mathrm{SN}$ photosphere (radius $\sim 10^{15} \mathrm{~cm}$ ) after maximum light and thus could not be responsible for an absorption line as deep as that observed in Figure 3. In order to produce a covering fraction closer to unity, there must either be large numbers of small clumps, or perhaps a larger sheet of material with perpendicular dimensions of $\sim 10^{17} \mathrm{~cm}$ (the rather high densities involved probably rule out the full spherical shell model proposed by Patat et al. 2007a). The total CSM mass along the line of sight (presumably more CSM is present in other directions) would then be in the range of $10^{-5}-10^{-2} M_{\odot}$ depending on the geometry.

Is it plausible for such a cloud (or clouds) to exist in the SN progenitor system? The measured velocity of the variable absorbing component is $2123 \mathrm{~km} \mathrm{~s}^{-1}$, while the local host galaxy ISM velocity near the position of the $\mathrm{SN}$ is $\approx 2130 \mathrm{~km}$ $\mathrm{s}^{-1}$. Assuming that the velocity measured for the local ISM is representative of the progenitor system, then the variable absorption component has a line-of-sight velocity of $\sim 10 \mathrm{~km}$ $\mathrm{s}^{-1}$ relative to the progenitor itself. At a constant velocity of $10 \mathrm{~km} \mathrm{~s}^{-1}$, material starting near the center of the progenitor system would take $\sim 10^{4}$ yr to reach a radius of $0.1 \mathrm{pc}$, but factoring in the gravitational deceleration the actual travel time would be $\sim 3000 \mathrm{yr}$ if the material originated in the red giant wind or $\sim 10 \mathrm{yr}$ if it was blown off from the white dwarf (which requires a much higher initial velocity). Regardless, this timescale is certainly far shorter than the age of the progenitor system or its evolutionary timescale, so producing CSM at such distances does not appear to be problematic. The survival of dense clumps similar to what we are proposing here, though, may be an issue: the thermal pressure in such a cloud is quite large, suggesting that the cloud should expand and dissipate on timescales of decades unless the average CSM pressure is also very high. We do not currently have enough information to determine whether such clumps of material could form from the wind of the companion alone, the interaction of the wind with surrounding material, or a nova eruption on the surface of the white dwarf. 

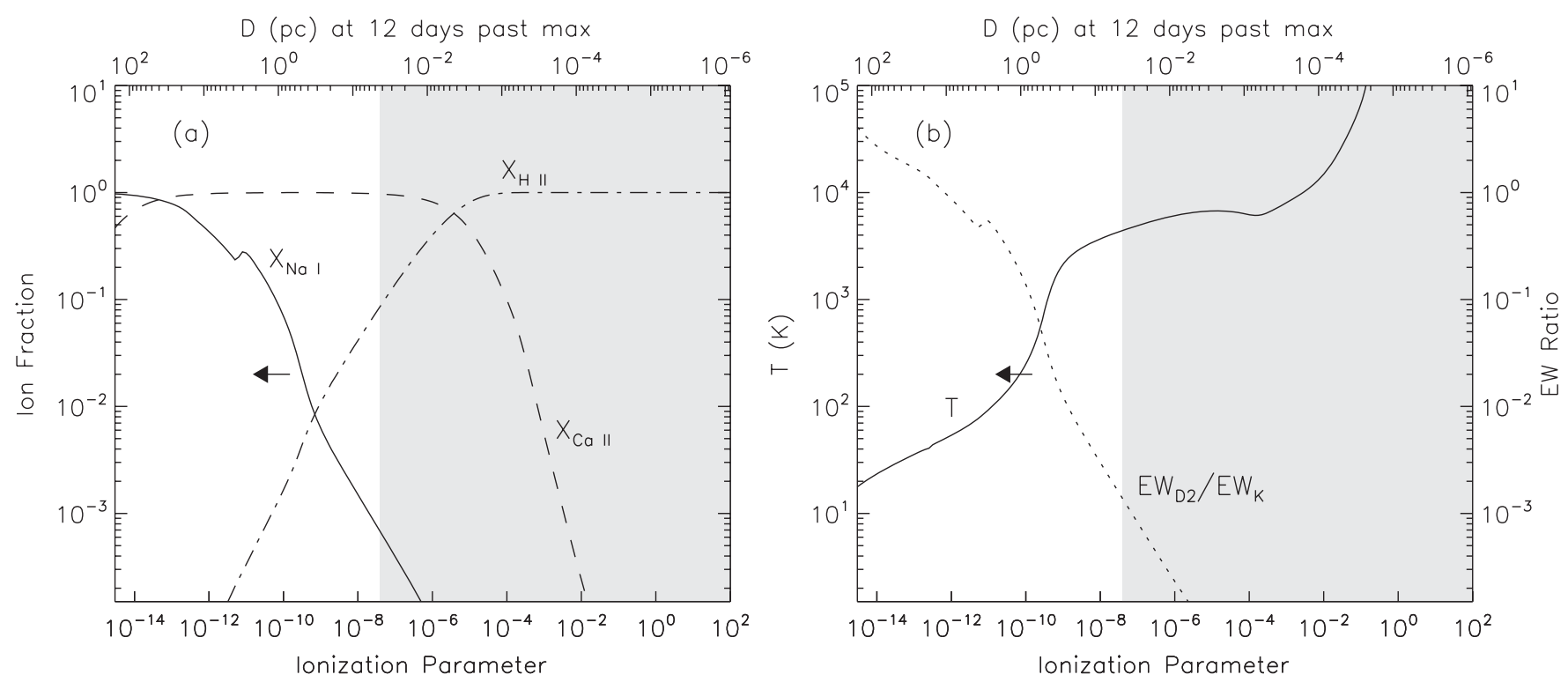

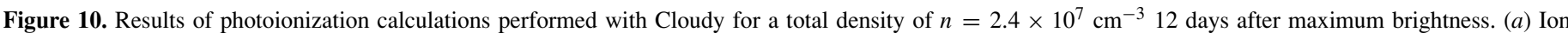

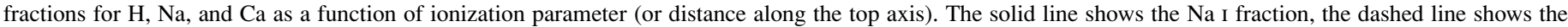

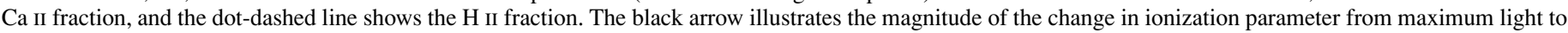

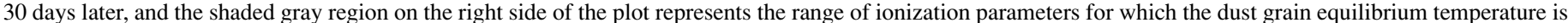

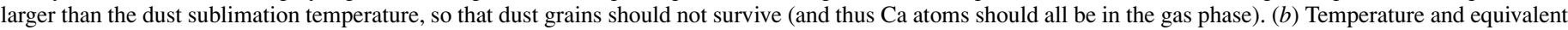

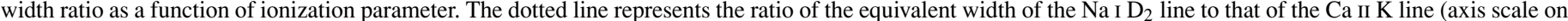

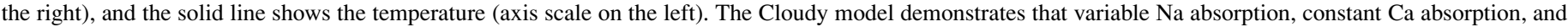
highly depleted $\mathrm{Ca}$ abundances can be obtained for distances larger than $\sim 0.1 \mathrm{pc}$ away from the site of the explosion.

\section{SUMMARY AND CONCLUSIONS}

We have presented a series of high-resolution spectra of the nearby SN Ia SN 2007le, a normal SN Ia in the subgroup that exhibits broad lines and high velocity gradients, similar to SN 2002bo and SN 2006X. The spectra reveal a complex set of absorption systems in the $\mathrm{Na} \mathrm{D}$ and $\mathrm{Ca} \mathrm{H} \& \mathrm{~K}$ lines. Our observations demonstrate that one of the $\mathrm{Na}$ absorption components changes systematically with time, increasing its equivalent width by $85 \mathrm{~m} \AA$ from maximum light to three months later. SN 2007le is the third SN Ia in which this effect has been seen, after SN 2006X (Patat et al. 2007a) and SN 1999cl (Blondin et al. 2009). As in SN 2006X, we detect no variations in the Ca II absorption column over the course of our observations.

In the initial study of SN 2006X, Patat et al. (2007a) modeled the varying absorption as occurring in several shells of circumstellar gas resulting either from the stellar wind of the companion to the SN progenitor (presumed to be a red giant) or recurrent nova eruptions. Subsequently, Chugai (2008) argued that the red giant wind scenario could only reproduce the observed behavior with an unrealistically high wind density. Chugai (2008) did note that a red giant wind plus a dense clump or shell of material might be compatible with the observed Na I and $\mathrm{Ca}$ II optical depths, but concluded that this solution was unlikely because of the difficulty of creating a sufficiently dense shell. Moreover, both SN 2006X and SN 1999cl were extremely heavily reddened, suggesting the possibility of a connection between variable absorption and properties of the interstellar medium along the line of sight. The much lower extinction toward SN 2007le demonstrates that variable absorption also occurs in SNe that are not seen through an unusually dense, dusty ISM. Conversely, it is now also clear that some highly obscured objects such as SN 2003cg (Blondin et al. 2009) and SN 2008fp (F. Patat et al. 2009, in preparation) do not exhibit variable absorption. We therefore carried out further photoionization calculations to investigate whether circumstellar material could explain the varying $\mathrm{Na}$ and constant $\mathrm{Ca}$ absorption profiles. Using a synthetic UV spectrum for a SN Ia, we found that a small $\left(10^{14} \mathrm{~cm}\right)$, dense $\left(10^{7} \mathrm{~cm}^{-3}\right)$ clump or clumps of material located $\sim 0.1 \mathrm{pc}$ away from the explosion can provide the necessary column density and ionization changes with time to account for the observations. In order to reproduce the observed $\mathrm{Na}$ I to Ca II EW ratio, this model requires a very large fraction of the $\mathrm{Ca}$ atoms to be depleted onto dust grains. The most likely origin for gas so close to the $\mathrm{SN}$ is that it was produced in the progenitor system. If this explanation is correct, the results indicate a single-degenerate progenitor system for SN 2007le, in accord with previous suggestions for SN 2006X.

Through the work of Blondin et al. (2009) and our own highresolution spectroscopy of additional $\mathrm{SNe}$ Ia (to be described in future papers), it is clear that the fraction of SN Ia events that show the variable Na signature is relatively small (as predicted by Patat et al. 2007a, 2007b for the recurrent nova scenario). In light of this rarity, it is noteworthy that all three of the $\mathrm{SNe}$ displaying variable absorption share common features in their early-time and maximum-light spectra, classifying them in the broad line group according to Branch et al. (2009) and the high velocity gradient group of Benetti et al. (2005, although the HVG classification of SN 2007le is less firm). With these groups comprising $\sim 30 \%$ of SNe Ia, the odds of three SNe exhibiting both variable absorption and BLs/high velocity gradients by chance (i.e., if there is no physical connection between the two) is low. Our observations of SN 2007le therefore support earlier speculations of a relationship between high velocity gradients and variable $\mathrm{Na}$ absorption.

These results still leave open the three explanations for variable absorption proposed in the literature: (1) SNe with high velocity gradients and variable absorption occur in a distinct progenitor system or with a different explosion mechanism than other SNe Ia, (2) SNe with high velocity gradients and 
variable absorption occur in the same progenitor systems as other SNe Ia, but with a peculiar geometry that yields higher observed velocities and makes variable absorption more likely, or (3) variable absorption is simply a line-of-sight effect that is independent of the properties of the SN itself. If the variable absorption originates in the ISM, then it is much more likely to be observed for objects that are seen through large ISM columns. The low extinction of SN 2007le, suggesting that it is not obscured by a substantial amount of ISM material, renders the third possibility less likely. Further high-resolution spectroscopy of BL/HVG SNe will be needed to continue investigating the first two scenarios and reveal whether high velocity gradients are a sufficient condition for variable $\mathrm{Na}$ absorption or merely a necessary one. It is also worth noting that in all three SNe with variable absorption, the absorption lines are systematically blueshifted with respect to the local ISM velocity, further pointing to a connection between the absorbing material and the $\mathrm{SNe}$ themselves.

In addition to variable $\mathrm{Na}$ absorption, we also detect $\mathrm{H} \alpha$ emission in the spectrum of SN 2007le, possibly originating from the $\mathrm{SN}$ itself. The narrow $\mathrm{H} \alpha$ emission line (FWHM $=25 \mathrm{~km} \mathrm{~s}^{-1}$ ) is spatially unresolved, coincident with the position of the $\mathrm{SN}$, and at a slightly different velocity than the surrounding diffuse interstellar emission. The amount of hydrogen that would need to be present in the circumstellar environment to produce the observed emission luminosity is $\sim 10^{-2}-10^{-3} M_{\odot}$. This feature is present at all high-resolution spectroscopic epochs, but as a result of its small equivalent width is not visible in any of the low-resolution spectra. The emission-line flux is constant within the uncertainties through 12 days after maximum light, but possibly strengthens in the observations obtained several months later. In the best of our spectra, $\mathrm{H} \beta,[\mathrm{N}$ II], and [S II] lines are also detected, but interestingly [O III] is not, suggesting a very low excitation for the ionized gas. If the increase in the $\mathrm{H} \alpha$ flux with time is real, then the emitting gas must be associated with the $\mathrm{SN}$, but further observations at late times after the SN has faded will be needed to confirm or reject this possibility.

The authors wish to acknowledge the very significant cultural role and reverence that the summit of Mauna Kea has always had within the indigenous Hawaiian community. We are most fortunate to have the opportunity to conduct observations from this mountain. The HET is named in honor of its principal benefactors, William P. Hobby and Robert E. Eberly. We thank Xavier Prochaska for his work on developing the HIRES data reduction pipeline and answering all of our extensive questions about it. We also thank the anonymous referee for constructive suggestions and acknowledge helpful conversations with Chris Burns, Mark Phillips, Doug Leonard, and Juna Kollmeier. Some calculations described in this paper were performed with version 07.02 of Cloudy, last described by Ferland et al. (1998). This research has made use of NASA's Astrophysics Data System Bibliographic Services and the NASA/IPAC Extragalactic Database (NED), which is operated by the Jet Propulsion Laboratory, California Institute of Technology, under contract with the National Aeronautics and Space Administration.

J.D.S. acknowledges the support of a Millikan Fellowship provided by Caltech and a Vera Rubin Fellowship from the Carnegie Institution of Washington. A.G. acknowledges support by the Israeli Science Foundation; an EU Seventh Framework Programme Marie Curie IRG fellowship; the Ministry of Science, Culture \& Sport, Israel and the Ministry of Research, France; and the Benoziyo Center for Astrophysics, UK-
Weizmann fund, a research grant from the Peter and Patricia Gruber Awards, and the William Z. and Eda Bess Novick New Scientists Fund at the Weizmann Institute. R.Q. and J.C.W. are supported in part by NSF grant AST-0707769. A.V.F.'s supernova group at U.C. Berkeley is supported by NSF grant AST-0607485, US Department of Energy grant DE-FG0208ER41563, and the TABASGO Foundation. KAIT and its ongoing operation were made possible by donations from Sun Microsystems, Inc., the Hewlett-Packard Company, AutoScope Corporation, Lick Observatory, the NSF, the University of California, the Sylvia \& Jim Katzman Foundation, and the TABASGO Foundation. Supernova research at the Harvard College Observatory is supported in part by the NSF through AST0606772.

\section{Facilities: Keck:I (HIRES)}

\section{REFERENCES}

Afanasyev, V. L., Burenkov, A. N., Zasov, A. V., \& Silchenko, O. K. 1992, Astron. Zh., 69, 19

Aldering, G. 2005, New Astron. Rev., 49, 346

Aldering, G., et al. 2006, ApJ, 650, 510

Asplund, M., Grevesse, N., \& Sauval, A. J. 2005, in ASP Conf. Ser. 336, Cosmic Abundances as Records of Stellar Evolution and Nucleosynthesis, ed. T. G. Barnes, III \& F. N. Bash (San Francisco, CA: ASP), 25

Astier, P., et al. 2006, A\&A, 447, 31

Badenes, C., Borkowski, K. J., Hughes, J. P., Hwang, U., \& Bravo, E. 2006, ApJ, 645,1373

Badnell, N. R. 2006, ApJS, 167, 334

Benetti, S., et al. 2005, ApJ, 623, 1011

Benetti, S., Cappellaro, E., Turatto, M., Taubenberger, S., Harutyunyan, A., \& Valenti, S. 2006, ApJ, 653, L129

Benetti, S., et al. 2004, MNRAS, 348, 261

Blondin, S., Prieto, J. L., Patat, F., Challis, P., Hicken, M., Kirshner, R. P., Matheson, T., \& Modjaz, M. 2009, ApJ, 693, 207

Blondin, S., \& Tonry, J. L. 2007, ApJ, 666, 1024

Borkowski, K. J., Blondin, J. M., \& Reynolds, S. P. 2009, ApJ, 699, L64

Branch, D., Dang, L. C., \& Baron, E. 2009, PASP, 121, 238

Branch, D., et al. 2006, PASP, 118, 560

Brown, P. J., et al. 2009, AJ, 137, 4517

Chandrasekhar, S. 1931, MNRAS, 91, 456

Chugai, N. N. 2008, Astron. Lett., 34, 389

Cox, N. L. J., \& Patat, F. 2008, A\&A, 485, L9

Crotts, A. P. S., \& Yourdon, D. 2008, ApJ, 689, 1186

de Vaucouleurs, G., \& Corwin, H. G., Jr. 1985, ApJ, 295, 287

Draine, B. T., \& Salpeter, E. E. 1979, ApJ, 231, 438

Elias-Rosa, N., et al. 2008, MNRAS, 384, 107

Ferland, G. J., Korista, K. T., Verner, D. A., Ferguson, J. W., Kingdon, J. B., \& Verner, E. M. 1998, PASP, 110, 761

Fesen, R. A., Saken, J. M., \& Hamilton, A. J. S. 1989, ApJ, 341, L55

Filippenko, A. V., Li, W. D., Treffers, R. R., \& Modjaz, M. 2001, in ASP Conf. Ser. 246, Small Telescope Astronomy on Global Scales, ed. W. P. Chen, C. Lemme, \& B. Paczyński (San Francisco, CA: ASP), 121

Filippenko, A. V., Silverman, J. M., Foley, R. J., Modjaz, M., Papovich, C., Willmer, C. N. A., Blondin, S., \& Brown, W. 2007, CBET 1101, 1

Foley, R. J., et al. 2003, PASP, 115, 1220

Gonzalez-Hernández, J. I., Ruiz-Lapuente, P., Filippenko, A. V., Foley, R. J., Gal-Yam, A., \& Simon, J. D. 2009, ApJ, 691, 1

Hamuy, M., Phillips, M. M., Suntzeff, N. B., Schommer, R. A., Maza, J., \& Aviles, R. 1996, AJ, 112, 2391

Hamuy, M., et al. 2003, Nature, 424, 651

Herbig, G. H. 1968, Z. Angew. Phys., 68, 243

Hicken, M., Wood-Vasey, W. M., Blondin, S., Challis, P., Jha, S., Kelly, P. L., Rest, A., \& Kirshner, R. P. 2009, ApJ, 700, 1097

Horne, K. 1986, PASP, 98, 609

Howard, W. E., III, Wentzel, D. G., \& McGee, R. X. 1963, ApJ, 138, 988

Howell, D. A. 2001, ApJ, 554, L193

Howell, D. A., et al. 2005, ApJ, 634, 1190

Hughes, J. P., Chugai, N., Chevalier, R., Lundqvist, P., \& Schlegel, E. 2007, ApJ, 670,1260

Iben, I., Jr., \& Tutukov, A. V. 1984, ApJS, 54, 335

Ihara, Y., Ozaki, J., Doi, M., Shigeyama, T., Kashikawa, N., Komiyama, K., \& Hattori, T. 2007, PASJ, 59, 811 
Immler, S., et al. 2006, ApJ, 648, L119

Jha, S., Riess, A. G., \& Kirshner, R. P. 2007, ApJ, 659, 122

Kennicutt, R. C., Jr., Bresolin, F., \& Garnett, D. R. 2003, ApJ, 591, 801

Kerzendorf, W. E., Schmidt, B. P., Asplund, M., Nomoto, K., Podsiadlowski, P., Frebel, A., Fesen, R. A., \& Yong, D. 2009, ApJ, in press (arXiv:0906.0982) Koribalski, B. S., et al. 2004, AJ, 128, 16

Krause, O., Tanaka, M., Usuda, T., Hattori, T., Goto, M., Birkmann, S., \& Nomoto, K. 2008, Nature, 456, 617

Leonard, D. C. 2007, ApJ, 670, 1275

Li, W. D., et al. 2000, in AIP Conf. Ser. 522, Cosmic Explosions, ed. S. S. Holt \& W. W. Zhang (New York: AIP), 103

Li, W., Filippenko, A. V., Treffers, R. R., Riess, A. G., Hu, J., \& Qiu, Y. 2001, ApJ, 546, 734

Li, W., et al. 2001, PASP, 113, 1178

Li, W., et al. 2003, PASP, 115, 453

Magrini, L., Vílchez, J. M., Mampaso, A., Corradi, R. L. M., \& Leisy, P. 2007, A\&A, 470, 865

Mannucci, F., Della Valle, M., Panagia, N., Cappellaro, E., Cresci, G., Maiolino, R., Petrosian, A., \& Turatto, M. 2005, A\&A, 433, 807

Mannucci, F., Della Valle, M., \& Panagia, N. 2006, MNRAS, 370, 773

Marietta, E., Burrows, A., \& Fryxell, B. 2000, ApJS, 128, 615

Matheson, T., Filippenko, A. V., Ho, L. C., Barth, A. J., \& Leonard, D. C. 2000, AJ, 120, 1499

Matheson, T., Filippenko, A. V., Li, W., Leonard, D. C., \& Shields, J. C. 2001, AJ, 121,1648

Mattila, S., Lundqvist, P., Sollerman, J., Kozma, C., Baron, E., Fransson, C., Leibundgut, B., \& Nomoto, K. 2005, A\&A, 443, 649

Mazzali, P. A., Chugai, N., Turatto, M., Lucy, L. B., Danziger, I. J., Cappellaro, E., della Valle, M., \& Benetti, S. 1997, MNRAS, 284, 151

Mazzali, P. A., et al. 2005a, ApJ, 623, L37

Mazzali, P. A., Benetti, S., Stehle, M., Branch, D., Deng, J., Maeda, K., Nomoto, K., \& Hamuy, M. 2005b, MNRAS, 357, 200

Miller, J. S., \& Stone, R. P. S. 1993, Lick Obs. Tech. Rep. 66

Monard, L. A. G., Yamaoka, H., \& Itagaki, K. 2007, CBET 1100, 1

Moore, C. E., Minnaert, M. G. J., \& Houtgast, J. 1966, National Bureau of Standards Monograph (Washington, DC: US Government Printing Office (USGPO))

Morton, D. C. 1975, ApJ, 197, 85

Murray, N., Martin, C. L., Quataert, E., \& Thompson, T. A. 2007, ApJ, 660 211

Nugent, P., Baron, E., Hauschildt, P. H., \& Branch, D. 1995, ApJ, 441, L33

Oemler, A., Jr., \& Tinsley, B. M. 1979, AJ, 84, 985

Oke, J. B., et al. 1995, PASP, 107, 375
Pakmor, R., Röpke, F. K., Weiss, A., \& Hillebrandt, W. 2008, A\&A, 489, 943

Panagia, N., Van Dyk, S. D., Weiler, K. W., Sramek, R. A., Stockdale, C. J., \& Murata, K. P. 2006, ApJ, 646, 369

Patat, F., et al. 2007a, Science, 317, 924

Patat, F., et al. 2007b, A\&A, 474, 931

Poole, T. S., et al. 2008, MNRAS, 383, 627

Prieto, J. L., et al. 2007, AJ, submitted (arXiv:0706.4088)

Quimby, R., Höflich, P., \& Wheeler, J. C. 2007, ApJ, 666, 1083

Riess, A. G., et al. 2007, ApJ, 659, 98

Ruiz-Lapuente, P. 2004, ApJ, 612, 357

Ruiz-Lapuente, P., et al. 2004, Nature, 431, 1069

Sauer, D. N., et al. 2008, MNRAS, 391, 1605

Scannapieco, E., \& Bildsten, L. 2005, ApJ, 629, L85

Schlegel, D. J., Finkbeiner, D. P., \& Davis, M. 1998, ApJ, 500, 525

Simon, J. D., et al. 2007, ApJ, 671, L25

Spitzer, L. J. 1954, ApJ, 120, 1

Spitzer, L. J. 1978, Physical Processes in the Interstellar Medium (New York: Wiley Interscience)

Stetson, P. B. 1987, PASP, 99, 191

Sullivan, M., et al. 2006, ApJ, 648, 868

Tanaka, M., et al. 2008, ApJ, 677, 448

Totani, T., Morokuma, T., Oda, T., Doi, M., \& Yasuda, N. 2008, PASJ, 60, 1327

Trundle, C., Kotak, R., Vink, J. S., \& Meikle, W. P. S. 2008, A\&A, 483, L47

Tull, R. G. 1998, Proc. SPIE, 3355, 387

van Hoof, P. A. M., Weingartner, J. C., Martin, P. G., Volk, K., \& Ferland, G. J. 2004, MNRAS, 350, 1330

Verner, D. A., Ferland, G. J., Korista, K. T., \& Yakovlev, D. G. 1996, ApJ, 465, 487

Vogt, S. S., et al. 1994, Proc. SPIE, 2198, 362

Wade, R. A., \& Horne, K. 1988, ApJ, 324, 411

Wang, L., Baade, D., \& Patat, F. 2007, Science, 315, 212

Wang, L., Baade, D., Patat, F., \& Wheeler, J. C. 2006, CBET 396, 2

Wang, L., \& Wheeler, J. C. 2008, ARA\&A, 46, 433

Wang, X., et al. 2008a, ApJ, 675, 626

Wang, X., Li, W., Filippenko, A. V., Foley, R. J., Smith, N., \& Wang, L. 2008b, ApJ, 677, 1060

Wang, X., Li, W., Filippenko, A. V., Foley, R. J., Smith, N., \& Wang, L. 2009, ApJ, 697, 380

Webbink, R. F. 1984, ApJ, 277, 355

Welty, D. E., \& Fitzpatrick, E. L. 2001, ApJ, 551, L175

Whelan, J., \& Iben, I. J. 1973, ApJ, 186, 1007

Williams, R., Mason, E., Della Valle, M., \& Ederoclite, A. 2008, ApJ, 685, 451

Wood-Vasey, W. M., et al. 2007, ApJ, 666, 694 